\title{
Sarcoglycanopathies: molecular pathogenesis and therapeutic
} prospects

\section{Dorianna Sandonà ${ }^{1}$ and Romeo Betto ${ }^{2, *}$}

Sarcoglycanopathies are a group of autosomal recessive muscle-wasting disorders caused by genetic defects in one of four cell membrane glycoproteins, $\alpha-, \beta-, \gamma$ - or $\delta$-sarcoglycan. These four sarcoglycans form a subcomplex that is closely linked to the major dystrophin-associated protein complex, which is essential for membrane integrity during muscle contraction and provides a scaffold for important signalling molecules. Proper assembly, trafficking and targeting of the sarcoglycan complex is of vital importance, and mutations that severely perturb tetramer formation and localisation result in sarcoglycanopathy. Gene defects in one sarcoglycan cause the absence or reduced concentration of the other subunits. Most genetic defects generate mutated proteins that are degraded through the cell's quality control system; however, in many cases, conformational modifications do not affect the function of the protein, yet it is recognised as misfolded and prematurely degraded. Recent evidence shows that misfolded sarcoglycans could be rescued to the cell membrane by assisting their maturation along the ER secretory pathway. This review summarises the etiopathogenesis of sarcoglycanopathies and highlights the quality control machinery as a potential pharmacological target for therapy of these genetic disorders.

Sarcoglycanopathies are autosomal recessive muscle-wasting disorders that result from genetic defects of four transmembrane glycoproteins, $\alpha-, \beta-, \gamma-$ and $\delta$-sarcoglycan. These four subunits form a distinct complex at the cell membrane of skeletal and cardiac muscle. The sarcoglycan complex, whose role is only now being revealed, is a component of a major complex formed by dystrophin at costameres in the cell membrane (Refs 1, 2, 3, 4). Sarcoglycanopathies are included in a large group of limb-girdle muscular dystrophy

${ }^{1}$ Department of Biomedical Sciences, University of Padova, 35121 Padova, Italy.

${ }^{2}$ C.N.R. Institute of Neuroscience, Neuromuscular Biology and Physiopathology, 35121 Padova, Italy.

*Corresponding author: Romeo Betto, C.N.R. Institute of Neuroscience, Neuromuscular Biology and Physiopathology, Viale Giuseppe Colombo 3, 35121 Padova, Italy. Tel: +39 049 8276027; Fax: +39049 8276040; E-mail: romeo.betto@bio.unipd.it

Accession information: doi:10.1017/S1462399409001203; Vol. 11; e28; September 2009 (c) Cambridge University Press 2009. Re-use permitted under a Creative Commons Licence-by-nc-sa. 
(LGMD) because these progressive muscle disorders predominantly affect proximal muscles around the scapular and the pelvic girdles. Mutations in individual sarcoglycans are responsible for LGMD-2C ( $\gamma$-sarcoglycan), LGMD-2D ( $\alpha$-sarcoglycan), LGMD-2E ( $\beta$ sarcoglycan) and LGMD-2F ( $\delta$-sarcoglycan) (Refs 5, 6, 7, 8, 9). The clinical phenotype of sarcoglycanopathies is very heterogeneous, and age of onset, rate of progression and severity can vary between and within affected families (Ref. 10). In general, the disease is characterised by progressive weakness and degeneration of skeletal muscle, leading to loss of ambulation, difficulties in breathing and often premature death. The majority of sarcoglycanopathies are associated with missense mutations that generate substitution of single residues that could lead to a misfolded protein. Analysis of muscle samples from patients showed that these mutations result in either the complete absence or the presence of only trace amounts of the protein in the cell membrane. Sarcoglycans are transmembrane proteins that mature in the endoplasmic reticulum (ER) where nascent proteins reach their native conformation through the activity of an efficient quality control system. Misfolded proteins are identified by the quality control system and retrotranslocated to the cytosol for proteasomal degradation through the ER-associated protein degradation (ERAD) pathway (Refs 11, 12). Occasionally, defective proteins may pass the quality control and reach the cell membrane, where, since they are nonfunctional and thus unstable, they are dismantled and degraded. Defects in each sarcoglycan have destabilising consequences on the entire sarcoglycan complex. Therefore, the pathogenetic mechanisms may comprise: (1) the processing of defective sarcoglycan subunits; (2) the inability to assemble into a complete complex; or (3) the targeting of a dysfunctional sarcoglycan complex to the cell membrane. Sequence analysis of sarcoglycans indicated that although many missense mutations might not have functional consequences, they are intercepted by the quality control system, which significantly slows their processing and results in the disposal of the mutant protein. Recent evidence shows that these misfolded 'functional' sarcoglycans could be rescued to the cell membrane by assisting them in the maturation process along the ER secretory pathway (Refs 13, 14). The purpose of this review is to provide a comprehensive overview of the molecular organisation of the sarcoglycan complex and of the pathogenetic events causing the disease. Novel therapeutic strategies for the treatment of sarcoglycanopathies based on interference of the ER-processing machinery are also evaluated.

\section{The dystrophin-glycoprotein complex}

The four sarcoglycans form a tetrameric subcomplex within the multimeric complex based on dystrophin in the cell membrane of skeletal and cardiac muscle. Dystrophin is a large actin-binding cytoskeletal protein that is essential for the organisation of the cellmembrane-associated dystrophin-glycoprotein complex (DGC). The DGC is enriched in costameres, which are specialised cytoskeletal structures connecting the plasma membrane and $\mathrm{Z}$ discs of peripheral myofibrils (Refs 2,15). Dystrophin is composed of four distinct functional domains: the N-terminal actinbinding domain, a long central domain containing 24 spectrin-like repeats, a cysteinerich domain and the C-terminal domain. The protein forms a tight link with the actin cytoskeleton through the N-terminal domain, an association reinforced by additional binding sites within some spectrin repeats (Ref. 1). At the distal region of the protein, dystrophin forms, via its cysteine-rich domain, a tight association with the transmembrane protein $\beta$-dystroglycan, which in turn is strongly linked to the extracellular $\alpha$-dystroglycan (Fig. 1). The two dystroglycans are products of a single gene that is post-translationally cleaved into two subunits (Ref. 17). The highly glycosylated $\alpha-$ dystroglycan forms a strong interaction with laminin $\alpha 2$, a major constituent of muscle fibre basement membrane. Thus, the backbone formed by dystrophin, dystroglycans and laminin $\alpha 2$ represents a transmembrane structure directly connecting the actin cytoskeleton to the extracellular matrix. The localisation of DGC in line with costameres is assured by the association of $\beta$-dystroglycan and dystrophin to two ankyrins (B and G), which are components of the subsarcolemmal cytoskeleton (Ref. 18). The DGC is thus thought to provide structural support to the plasma membrane and to protect it from the 
Figure 1. The dystrophin-glycoprotein complex. Simplified scheme of dystrophin-glycoprotein complex (DGC) organisation. Dystrophin is localised in the cytoplasmic face of skeletal and cardiac cell membranes. Dystrophin binds actin filaments through two specific binding sites in its C-terminal domain and sites in the spectrin-like-repeat portion. The cysteine-rich domain assures the binding of dystrophin to the transmembrane $\beta$-dystroglycan, which in turn associates extracellularly with $\alpha$-dystroglycan (blue box). $\alpha$ dystroglycan interacts with laminin $\alpha 2$ and other cell matrix components, completing the backbone of the DGC. The sarcoglycan-sarcospan complex (light blue box), forms a lateral association with dystroglycans. $\mathrm{N}$-glycan and O-glycan indicate, respectively, the $\mathrm{N}$ - and O-glycosylation moieties post-translationally added to sarcoglycans and dystroglycans. Additional sarcoglycan partners have been proposed (not all indicated), both intracellularly and extracellularly. Dystrobrevin, syntrophin (Syn) and neuronal nitric oxide synthase (nNOS) are intracellular components of the DGC. Many other proteins have been indicated to interact with DGC, either permanently or dynamically; a few of these are indicated. For a complete list of DGC components the reader should refer Refs 1, 2, 15, 16.

mechanical stress of contractile activity by transmitting the lateral tension generated by muscle contraction to the extracellular matrix (Refs 15, 19). The critical protective role of dystrophin and of the entire membraneassociated DGC is demonstrated by the fact that genetic defects of dystrophin are responsible for the most common type of muscular dystrophy, the incurable Duchenne muscular dystrophy
(DMD) and the milder Becker muscular dystrophy (BMD), whereas defects of laminin $\alpha 2$ and $\alpha$-dystroglycan glycosylating enzymes cause diverse forms of muscular dystrophy (Refs 2, 20, 21). With a defective DGC, the backbone structure is dismantled so that the cell membrane becomes exposed to muscle contraction stresses. As a consequence, cell membrane focal ruptures might occur, leading 
to transient intracellular calcium influx, which triggers a series of pathogenic events that result in muscle degeneration and the dystrophic phenotype (Refs 2, 21).

\section{Role of sarcoglycans in the dystrophin- glycoprotein complex}

Loss of sarcoglycan is the specific cause of sarcoglycanopathy, whereas the absence of dystrophin in DMD causes instability of the complex and also leads to the severe reduction of sarcoglycans. Thus, an altered sarcoglycan complex is a common trait of the two muscular dystrophies and probably represents an aggravating condition in DMD.

The role of the four sarcoglycans in the molecular organisation of the DGC is not yet well defined. The sarcoglycan complex is known to form a tight side-association with dystroglycan (Refs 22, 23), but is also involved in composite molecular relationships with other constitutive DGC elements such as $\alpha$ dystrobrevin and syntrophin (Ref. 24), neuronal nitric oxide synthase (nNOS) (Ref. 25) and sarcospan (Ref. 26) (Fig. 1). Sarcospan is an integral component of DGC, which directly interacts with and stabilises sarcoglycans. The relevance of this transmembrane protein is, however, still uncertain, because ablation of the sarcospan gene in mice does not result in myopathy (Ref. 27). The sarcoglycan complex, excluding $\gamma$-sarcoglycan, is thought to form an association with $\alpha$-dystroglycan (Ref. 23 ) via an extracellular proteoglycan, biglycan (Ref. 28). The intracellular tail of $\beta$ - and $\delta$-sarcoglycan seems to associate directly with the $\mathrm{C}$-terminus of dystrophin (Ref. 29), whereas the N-terminal region of $\alpha$-dystrobrevin secures sarcoglycans to dystrophin (Ref. 24). $\alpha$-Dystrobrevins, syntrophin and nNOS form a signalling subunit associated with dystrophin through the Cterminal domain of dystrobrevin. Additional proteins seem to interact with sarcoglycans at the cell membrane. Two hybrid screens identified $\gamma$-filamin as a $\gamma$ - and $\delta$-sarcoglycaninteracting protein (Ref. 30) and, as a result, $\gamma$ filamin is reduced in LGMD-2C ( $\gamma$-sarcoglycan) but not in LGMD-2D ( $\alpha$-sarcoglycan) (Ref. 31). Since $\gamma$-filamin is an actin-binding protein, it provides, through the interaction with the sarcoglycan complex, additional structural linkages between the DGC and the actin cytoskeleton. The precise physiological role of biglycan and $\gamma$-filamin, however, is not well understood. Recently, aquaporin 4, a musclespecific water channel, was shown to be associated with sarcoglycan through $\alpha 1$ syntrophin, an interaction validated by its severe reduction in muscles of sarcoglycanopathy patients (Ref. 32).

Altogether, these data demonstrate that the sarcoglycan complex forms multiple interactions with the other DGC components and enforce the view of its crucial role in stabilising the whole DGC structure. However, the sarcoglycans might also be involved in signal transduction (Refs 2, 3, $21,33)$, a role that is primarily supported by the anchorage provided by the complex to nNOS through dystrobrevin and syntrophin (Ref. 25). Moreover, the sarcoglycan complex has been shown to cooperate with integrins in mediating cell adhesion to the extracellular matrix (Ref. 34). In support of this, integrins are upregulated when the complex is missing (Ref. 35). The sarcoglycan complex also interacts with the 16 $\mathrm{kDa}$ subunit of vacuolar $\mathrm{H}^{+}$-ATPase, and thence with $\beta 1$-integrin (Ref. 36) - a finding that further supports bidirectional signalling with integrins (Ref. 34). Notably, it has been demonstrated that the cytoplasmic tail of $\gamma$ sarcoglycan is phosphorylated after mechanical stimulation, suggesting that it is a signalling sensor of contractility (Ref. 37). In addition, $\alpha$ sarcoglycan possesses an ATP-binding site, conferring ATP-hydrolysing activity to the protein (Refs 38, 39). This activity suggests a possible role in modulating the signalling initiated by extracellular nucleotides and a role in the extracellular ATP-dependent modulation of skeletal muscle contractility (Ref. 40). The critical role of the sarcoglycan complex, whether mechanical or signalling, is further corroborated by a recent study showing that the complete absence of the sarcoglycan complex in mice lacking both $\delta$-sarcoglycan and dystrophin exacerbates the pathological course (Ref. 41). This evidence also suggests that the residual expression of the sarcoglycan complex contributes to the milder phenotype of dystrophin-deficient $m d x$ mice compared with that of patients with DMD (Ref. 41).

\section{The sarcoglycan complex in sarcoglycanopathies}

Studies of patient muscle biopsies provide additional important information on the 
sarcoglycan complex and its contribution to the molecular organisation of the DGC. Analyses of muscle biopsies from sarcoglycanopathy patients demonstrated that the absence or reduced expression of one sarcoglycan has important, but variable consequences for the stability of the other remaining components at the cell membrane. In general, the most clinically severe course was observed in patients in which the involved sarcoglycan was absent, with a variable milder phenotype when residual protein is present. Early studies on patients affected by sarcoglycanopathies reported that mutations in one sarcoglycan gene led to the reduction or absence of the other sarcoglycans, with negligible consequences for the DGC (Refs 6, 7, 8, 42, 43, $44,45,46,47,48,49)$. By contrast, more recent evidence shows that the absence of individual sarcoglycans has indirect consequences on DGC stability. In LGMD-2E and -2F patients, defects in genes encoding $\beta$ - and $\delta$-sarcoglycan results in the absence of the four sarcoglycans from the plasma membrane and the reduction of dystrophin and dystroglycan (Refs 50, 51, 52, 53 , 54). In LGMD-2D patients, defects in $\alpha$ sarcoglycan causes the secondary loss of $\beta$ - and $\delta$-sarcoglycan and a severe reduction in $\gamma$ sarcoglycan, which can be absent altogether in more severely affected patients; $\beta$-dystroglycan and dystrophin were reduced in most cases (Refs 50, 52, 53, 54). In $\gamma$-sarcoglycan-deficient patients (LGMD-2C) trace amounts of residual sarcoglycans are evident at the cell membrane, whereas no consequences for the DGC or a reduction of dystrophin are reported (Refs 50, $52,53,54,55,56)$. The absence of the sarcoglycan complex in muscle of patients affected by these diseases is reported to expose $\beta$-dystroglycan to the activity of a matrix metalloproteinase, thus providing an explanation for its reduced level in sarcoglycanopathies (Ref. 57).

Animal models exist for each of the four sarcoglycanopathies and show similar results to the human situation. The absence of $\alpha-, \beta-$ and $\delta$-sarcoglycan causes the complete loss of the other three sarcoglycans and some destabilisation of dystroglycans (Refs 23, 58, 59, $60,61,62,63,64)$. By contrast, the absence of $\gamma$ sarcoglycan in mice weakens the stability of $\beta$ and $\delta$-sarcoglycan and causes severe reduction of $\alpha$-sarcoglycan, but the expression and localisation of dystrophin, dystroglycan and laminin- $\alpha 2$ are unaffected (Refs 62, 65, 66, 67). $\delta$-Sarcoglycan-deficient animals develop cardiomyopathy, which is rarely observed in LGMD-2F patients, with loss of the sarcoglycan complex even in smooth muscle (Ref. 60); $\beta$ and $\gamma$-sarcoglycan-deficient mice, but not $\alpha$ sarcoglycan-null mice, also display some cardiac involvement.

The sarcoglycan family of proteins in flies is simpler than in vertebrates: the fly genome encodes a single orthologue of vertebrate $\alpha$ and $\varepsilon$-sarcoglycans ( $\alpha \varepsilon$-sarcoglycan), a $\beta$ sarcoglycan and a single orthologue of $\gamma$ - and $\delta$-sarcoglycans ( $\gamma \delta$-sarcoglycan). In Drosophila, a tetrameric organisation of the complex is suggested, with the association of $\alpha \varepsilon$ sarcoglycan, $\beta$-sarcoglycan and two copies of $\gamma \delta$-sarcoglycan (Ref. 68). A $\gamma \delta$-sarcoglycandeficient model has been generated, which caused skeletal and cardiac defects (Ref. 69); however, the fate of the other sarcoglycans was not determined. Orthologues of sarcoglycan genes have also been identified in zebrafish, which represent an established model of vertebrate development (Ref. 70). Knockdown of $\delta$-sarcoglycan caused skeletal and cardiac development defects and reduced the levels of $\beta$ - and $\gamma$-sarcoglycan (Ref. 71).

In summary, muscle analysis of various LGMD patients and of related animal models shows that the absence of or defects in $\beta$ - and $\delta$-sarcoglycan has dramatic consequences on the expression of all other subunits, whereas defects in $\gamma$-sarcoglycan have the least effect on expression of the other sarcoglycans. Interestingly, the absence of $\alpha$-sarcoglycan in humans causes the absence or severe reduction of $\gamma$-sarcoglycan, but $\beta$ - and $\delta$-sarcoglycan are slightly spared. By contrast, in $\alpha$-sarcoglycandeficient mice, the other sarcoglycans are completely lost. Interestingly, expression of $\gamma$-sarcoglycan and, to a lesser extent, $\alpha$-sarcoglycan, is reduced more than $\beta$ - and $\delta$-sarcoglycan across all sarcoglycanopathies.

\section{The molecular organisation of the sarcoglycan complex}

Data from LGMD patients and related animal models demonstrate that the four sarcoglycans have an important role at the plasma membrane, but only when they exist as a complex. A better understanding of sarcoglycan

Accession information: doi:10.1017/S1462399409001203; Vol. 11; e28; September 2009 (c) Cambridge University Press 2009. Re-use permitted under a Creative Commons Licence-by-nc-sa. 
complex organisation, processing and function is thus central to improve our knowledge of the pathogenic mechanisms responsible for the dystrophic phenotype.

Sarcoglycans are single-pass transmembrane proteins, with a short intracellular tail and a large extracellular glycosylated portion that is rich in conserved cysteine residues. Six sarcoglycans have been cloned so far: $\alpha-, \beta-$, $\gamma^{-}, \delta-, \varepsilon-$ and $\zeta$-sarcoglycan (Table 1). $\alpha$ - and $\gamma$-sarcoglycan are expressed exclusively in skeletal and cardiac muscle, whereas $\varepsilon^{-}, \beta-, \delta-$ and $\zeta$-sarcoglycan are more widely distributed (Refs 5, 6, 7, 8, 9, 72, 73, 74). The $\alpha$ - and $\varepsilon$ sarcoglycans, are type I membrane proteins with an extracellular N-terminal domain, whereas $\beta-, \gamma-, \delta$ - and $\zeta$-sarcoglycans are type II membrane proteins, with an extracellular Cterminal domain. $\alpha$ - and $\varepsilon$-sarcoglycan are highly homologous, as are $\beta-, \gamma-, \delta$ - and $\zeta$ sarcoglycan, with the latter being functionally more similar to $\gamma$-sarcoglycan than to $\delta$ sarcoglycan (Ref. 75). A cysteine cluster is present in the extracellular domain of all sarcoglycans and is predicted to be important for the tertiary structure of the protein and for assembly of the whole complex (Ref. 22). All four sarcoglycans possess putative phosphorylation sites in the intracellular domain, indicating a possible post-translational modulation of the protein or complex.

Protein sequence analysis shows that $\alpha$ sarcoglycan contains an $\mathrm{N}$-terminal signal sequence, one transmembrane domain, five extracellular cysteines and two putative $\mathrm{N}$ linked glycosylation sites, which are conserved in all species (Table 1 and Fig. 5). Cleavage of the signal sequence and glycosylation generate a protein of about $50 \mathrm{kDa}$. In the $\mathrm{N}$-terminal portion, $\alpha$-sarcoglycan has a cadherin-like domain and $\mathrm{Ca}^{2+}$-binding pockets, which have also been described in $\alpha$-dystroglycan, suggesting a possible $\mathrm{Ca}^{2+}$-dependent heterotypic adhesion between the two proteins (Ref. 76). In addition, $\alpha$-sarcoglycan has a putative ATP-binding sequence in the extracellular domain, which is conserved in all species (Ref. 38). The predicted molecular mass of glycosylated $\beta$-sarcoglycan is about $43 \mathrm{kDa}$ and its large extracellular portion has five cysteine residues and three putative N-linked glycosylation sites. The mass of glycosylated $\gamma$ and $\delta$-sarcoglycan is $\sim 35 \mathrm{kDa}$. $\gamma$-sarcoglycan has four cysteine residues and one putative $\mathrm{N}$ linked glycosylation site in its extracellular portion, whereas $\delta$-sarcoglycan has four cysteine residues and three putative $\mathrm{N}$-linked glycosylation sites. The C-terminus of $\beta-, \delta$ - and $\gamma$-sarcoglycan has three conserved cysteine residues in a fixed position that is similar to those in epidermal growth factor (EGF), suggesting a receptor-like function for an unknown agonist (Refs 3, 29). It is worth noting that the presence of a putative N-linked glycosylation site in a protein does not necessarily equate to a real glycosylation event. In fact, although the molecular masses of unglycosylated $\gamma$ - and $\delta$-sarcoglycan are identical $(32 \mathrm{kDa})$, the presence of three $\mathrm{N}$ glycans in $\delta$-sarcoglycan is expected to generate a larger mass than that of $\gamma$-sarcoglycan, which has one N-linked glycosylation site. Therefore, either only one of the $\delta$-sarcoglycan $\mathrm{N}$-linked glycosylation sites is eventually glycosylated or the two proteins are modified differently upon post-translation processing.

It is widely assumed that sarcoglycans are organised at the cell membrane as a tetramer, in a complex that is exclusively expressed in striated muscles; this is consistent with the notion that mutations in $\alpha_{-}^{-}, \beta-, \gamma_{-}$and $\delta_{-}$ sarcoglycan are responsible for muscle sarcoglycanopathies. Mutations in the gene encoding $\varepsilon$-sarcoglycan are associated with myoclonus dystonia syndrome (Ref. 77), whereas no disease has been so far associated with mutations in the $\zeta$-sarcoglycan gene. Organisation of the sarcoglycan complex occurs in a strict equimolar stoichiometry (Ref. 47), although atypical organisation and localisation of sarcoglycans have also been reported. For example, in human smooth muscle, a pentameric or exameric sarcoglycan complex has been proposed (Ref. 78). Moreover, $\delta$ - and $\gamma$-sarcoglycan seem to have an additional intracellular membrane localisation, but any contribution to muscle physiology and/or pathology is unknown (Ref. 79).

\section{Assembly and trafficking of the sarcoglycan complex}

Studies on the assembly of the sarcoglycan complex during the early stage of myotube differentiation show that sarcoglycans are cotranslationally translocated in the ER, where they associate during transport through the

Accession information: doi:10.1017/S1462399409001203; Vol. 11; e28; September 2009 (c) Cambridge University Press 2009. Re-use permitted under a Creative Commons Licence-by-nc-sa. 
Table 1. Human sarcoglycan protein data

\begin{tabular}{|c|c|c|c|c|c|c|c|}
\hline Protein & Gene & Chromosome & Disease & $\mathbf{A A}$ & Mass $^{a}$ & $\mathbf{N}$ & C \\
\hline$\alpha$-Sarcoglycan & $S G C A$ & $17 q 21.33$ & LGMD-2D & 387 & 50 & 2 & 5 \\
\hline$\beta$-Sarcoglycan & SGCB & $4 q 11$ & LGMD-2E & 318 & 43 & 3 & 5 \\
\hline$\gamma$-Sarcoglycan & SGCG & $13 q 12$ & LGMD-2C & 291 & 35 & 1 & 4 \\
\hline 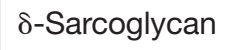 & $S G C D$ & $5 q 33.3$ & LGMD-2F & 290 & 35 & 3 & 4 \\
\hline$\varepsilon$-Sarcoglycan & SGCE & $7 q 21.3$ & MDS & 405 & 45 & 1 & 4 \\
\hline$\zeta$-Sarcoglycan & $S G C Z$ & 8p22 & Unknown & 299 & 35 & 1 & 4 \\
\hline
\end{tabular}

Golgi to the plasma membrane (Refs 22, 80) (Fig. 2).

In the ER, the quality control machinery facilitates polypeptide folding to ensure that nascent proteins assume the correct conformation, and, if necessary, it recycles the proteins until the native conformation is obtained (Fig. 3a-c). Glycosylation of proteins such as sarcoglycans is performed by an oligosaccharyltransferase, which attaches a composite oligosaccharide composed of three glucose, nine mannose and two n-acetyl glucosamine residues ( $\mathrm{N}$-glycan) to the nascent polypeptide. Subsequently, two glucoses are sequentially trimmed from the N-glycan by glucosidase I and II, while two lectin chaperons, calnexin and calreticulin, associate with the monoglycosylated proteins to attain their pre-native conformation (Refs 11, 12). Protein folding is then performed by oxidoreductases of the PDI family and peptidyl-propyl cis/trans isomerases, which facilitate the oxidative folding of the protein, formation and isomerisation of disulfide bonds, the correct cis or trans isomerisation and prevent the formation of aggregates (Ref. 12). At the end of the calnexin-calreticulin cycle, the enzyme UDP-glucose glycoprotein glucosyltransferase (UGGT) reattaches a glucose to imperfectly folded proteins, causing their reintroduction into the calnexin and calreticulin cycle (Ref. 81) (Fig. 3b). Properly folded proteins are ignored by UGGT and proceed along the maturation pathway (Fig. 3c). Export of native proteins occurs at ER-specific sites where budding of vesicles coated with coat protein complex II takes place and by which they enter the Golgi complex (Fig. 3d).

Trafficking of transmembrane multimeric complexes requires more elaborate processing because the individual membrane proteins are usually assembled in the ER and then transported as a unit to the Golgi and the cell membrane (Fig. 2). Evidence indicates that multimeric complexes may also encounter postER quality control checkpoints that monitor and assist the oligomerisation process, probably at ER-Golgi intermediate and cis-Golgi compartments (Ref. 11). Experimental evidence in $\mathrm{CHO}$ cells demonstrated that the simultaneous synthesis of the four sarcoglycans is mandatory for proper assembly and cell membrane localisation of the sarcoglycan complex (Ref. 82). Biochemical analyses in C2C12 myogenic cells showed that sarcoglycans associate to form a complex in the ER as soon as they are synthesised. Thereafter, the sarcoglycan complex associates with dystroglycans and sarcospan only during transportation from the Golgi to the cell membrane (Ref. 83). The latter finding implies that sarcoglycans, sarcospan and dystroglycan may exit the ER independently and then associate during transport to the cell membrane (Ref. 84) (Fig. 2). The association of the sarcoglycan complex with dystroglycans en route to the cell membrane probably favours the subsequent recruitment of cytosolic dystrophin once it reaches its destination. In primary mouse myotubes, it was demonstrated 


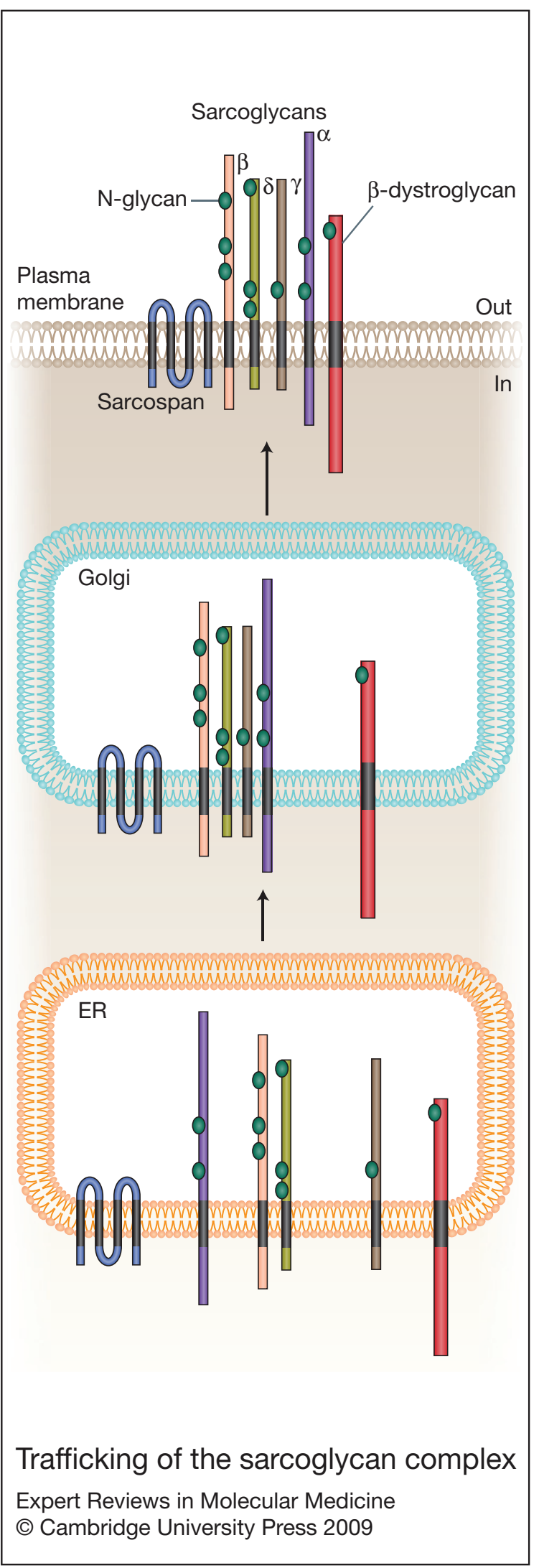

that $\beta-, \delta$ - and $\gamma$-sarcoglycans form a strong interaction, whereas $\alpha$-sarcoglycan seems to be less tightly associated (Ref. 22). The overall evidence indicates the following sequential events for sarcoglycan association: (1) $\beta$ sarcoglycan initiates a strong interaction with $\delta$ sarcoglycan; (2) $\gamma$-sarcoglycan is added to the $\beta \delta$-sarcoglycan core; (3) $\alpha$-sarcoglycan is recruited by interacting with $\gamma$-sarcoglycan (Refs 22, 85). An alternative association sequence has also been proposed, where $\alpha$ sarcoglycan directly interacts with the $\beta \delta$ sarcoglycan core (Refs 86, 87). An intriguing finding in COS-1 (Ref. 85) and in HEK293 (Ref. 86) cells shows that $\beta$ - and $\delta$-sarcoglycans might be transported to the cell membrane even when expressed without $\alpha$ - and $\gamma$-sarcoglycan. In any case, these data reinforce the view of the fundamental role of the $\beta \delta$-sarcoglycan core in the assembly process of the complex. However, the precise molecular interactions controlling assembly of the four sarcoglycans remain undefined.

A recent study in COS-1 cells identified novel functional domains in the extracellular portion of sarcoglycans that seem to regulate association and favour transport to the cell membrane. In particular, regions of the extracellular portion close to the transmembrane domain appear to be important for regulation of the interaction between $\beta$ - and $\delta$-sarcoglycan and $\alpha$ - and $\gamma$-sarcoglycan, whereas a more distal region seems to control the interaction between $\beta$ - and $\gamma$-sarcoglycan (Ref. 29). Moreover, these authors also demonstrate that $\mathrm{N}$-glycosylation and the cysteine-rich domain of $\delta$-sarcoglycan are

Figure 2. Trafficking of the sarcoglycan complex. The four sarcoglycans ( $\alpha, \beta, \gamma$ and $\delta$ ) are synthesised in the ER where they undergo cotranslational glycosylation (N-glycan) and proper conformational folding. Association of the four sarcoglycans occurs in the ER, with the $\beta \delta$ sarcoglycan core being the trigger for the assembly of the tetrameric complex. The complex is then transported to the plasma membrane through the Golgi system, where it most likely assembles with sarcospan and dystroglycan. Once in the cell membrane, the other cytoplasmic components of the dystrophinglycoprotein complex (DGC), aggregate to form the final structure. 


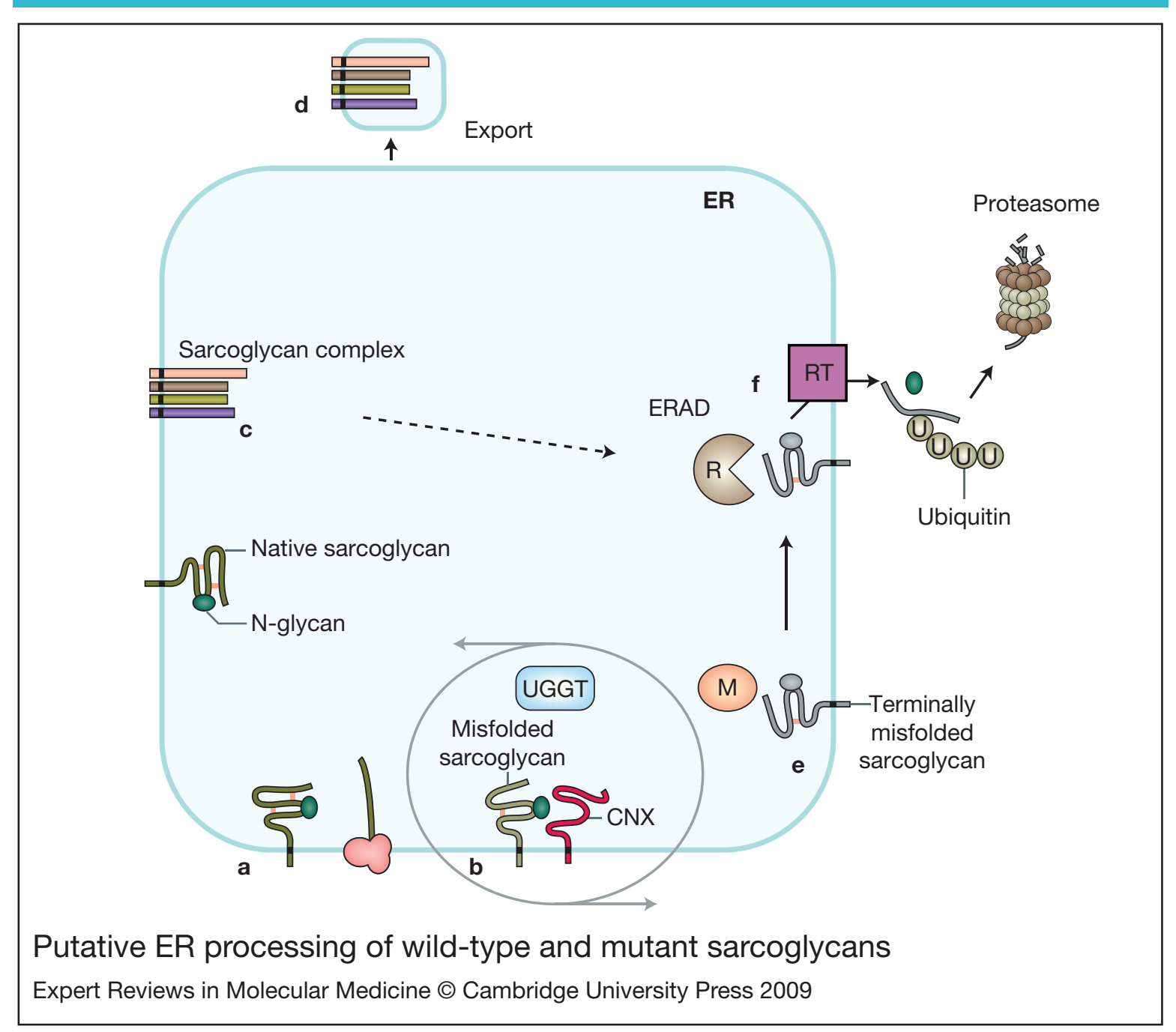

Figure 3. Putative ER processing of wild-type and mutant sarcoglycans. Nascent sarcoglycans elongate into the ER where they undergo cotranslational glycosylation and proper conformational folding (a). Non-native polypeptides may experience repetitive rounds of folding within the calnexin (CNX)-calreticulin cycling system, a process regulated by UDP-glucose glycoprotein glucosyltransferase (UGGT) (b). A successfully folded protein then enters the maturation process, which comprises assembly with the other sarcoglycans to form a tetrameric complex (c). The protein complex is then exported from the ER (d). Misfolded proteins are degraded by endoplasmic reticulum-associated degradation (ERAD) by intervention of mannosidase I (M) (e). Individual components not able to assemble are also degraded (dotted arrow). ERAD occurs in a composite process of recognition (R), targeting for ER retrotranslocation (RT), and degradation of the terminally misfolded protein by the ubiquitin-proteasome system (f).

crucial for the localisation of sarcoglycans to the cell membrane, and suggest that the intracellular tails of $\beta$ - and $\delta$-sarcoglycan interact with dystrophin (Ref. 29).

The individual heterologous expression of $\alpha$-sarcoglycan, but not of the other subunits, results in the localisation of the protein at the cell membrane even in the absence of the remaining sarcoglycans. Surprisingly, this occurs in cells of human origin, such as
HEK293 and HeLa cells (Refs 39, 88) but not in COS-1 cells from monkey or $\mathrm{CHO}$ cells from hamster (Refs 82, 85). Moreover, $\alpha$-sarcoglycan cell membrane localisation seems to be dynamic, because the protein recycles from the membrane to endosomes (Ref. 88). This evidence suggests that undefined sequence motifs of human $\alpha$-sarcoglycan might permit its preferential solitary trafficking to the cell membrane. Conversely, it is possible that the

Accession information: doi:10.1017/S1462399409001203; Vol. 11; e28; September 2009 (C) Cambridge University Press 2009. Re-use permitted under a Creative Commons Licence-by-nc-sa. 
difference might reside in the maturation, sorting and trafficking rules of the human protein-export apparatus. This possibility is validated by recent evidence showing that the R77C $\alpha$-sarcoglycan mutant, the most common LGMD-2D mutation, remains trapped in the ER when expressed in human cells, either alone or together with the other sarcoglycans (Refs 13, 14, 86). By contrast, in knock-in mice homozygous for the H77C mutation (in mice, $\mathrm{R}$ at position 77 is conservatively substituted by $\mathrm{H}$ ), $\alpha$-sarcoglycan and the entire complex is correctly localised at the cell membrane and mice did not develop muscular dystrophy (Refs 13, 89). Since no information on the tertiary structure of $\alpha$ sarcoglycan is available, it is possible that the protein region around $\mathrm{H} 77$ in mice might differ from that in humans such that this substitution could be considered a polymorphism in mice. Intriguingly, when human R77C $\alpha$-sarcoglycan is transfected into muscles of $\alpha$-sarcoglycanknockout mice some aggregates do form, but the majority reaches the cell membrane (Refs 13, 89).

\section{Pathogenetic mechanisms}

In sarcoglycanopathy, mutations in any of the four sarcoglycan genes cause a reduction or the absence of the defective protein, and secondarily affect expression of the other subunits. A reduction in protein levels can have several causes, such as defects that hamper gene expression or lead to aberrant maturation of the mRNA transcript, or defects in protein processing (Ref. 90), where its fate then depends on the ER quality control system. Complete absence of a protein can result from nonsense mutations, which instead of producing truncated sarcoglycans, trigger nonsense-mediated mRNA decay. Absence of a protein might also result from missense mutations that lead to production of misfolded proteins, which are then recognised and retained in the ER, and after unsuccessful cycling in the calnexin and calreticulin system, are ultimately degraded through the ubiquitinproteasome system (Fig. 3e, f). A mutant protein might evade the quality control mechanism, but since it will probably not be able to form a stable tetramer it will still be degraded (Fig. 3c, f). Finally, a mutant sarcoglycan protein might fold and form a tetramer, exit the ER and reach the cell membrane, where it could cause loss of function or instability of the entire sarcoglycan complex. In this case, the whole structure will be dismantled and undergo lysosomal-proteasomal degradation. The residual expression level of the remaining wildtype sarcoglycans probably depends on the association rules and trafficking possibilities described above. However, the presence of residual expression of a mutant protein in the cell membrane suggests that the mutant might escape the ER quality control system, at least in part, and forms a tetramer that is transported to the cell membrane. The resulting dystrophic phenotype might depend on insufficient levels of either the protein or the complete complex, or to loss-of-function consequences.

According to the Leiden Open Variation Database (Ref. 91) (http://www.lovd.org), and to recent evidence (Refs 53, 92), at least 176 sequence variants in the coding region of sarcoglycans have been so far reported to cause LGMD-2C to LGMD-2F. Seventy-one are mutations that generate a truncated or aberrant protein or lead to nonsense-mediated decay of the transcript, whereas 105 are missense mutations that cause protein misfolding. Usually, mutated misfolded proteins synthesised in the ER are intercepted, retrotranslocated in the cytosol and delivered for dismantling by the ubiquitin-proteasome through the multifaceted ERAD system (Fig. 4). Recognition of misfolded proteins is quite rapid, and proteins are delivered for degradation in a ubiquitin-dependent manner, without any detectable lag following their synthesis. The process is intended to prevent the accumulation of defective proteins and formation of toxic aggregates that might block trafficking in the secretory pathway. However, several misfolded proteins are reported to be prone to aggregation, as is the case for many myofibrillar myopathies (Ref. 93) and neurodegenerative disorders, such as Parkinson, Alzheimer and prion-associated diseases (Ref. 94).

Recently, two different groups have demonstrated the involvement of the cell quality control system in the pathogenesis of sarcoglycanopathies in heterologous cell models (Refs 13, 14). Both papers show that different $\alpha$-sarcoglycan mutants with single amino acid substitutions are ubiquitinated and rapidly degraded by the proteasome. Intriguingly, 


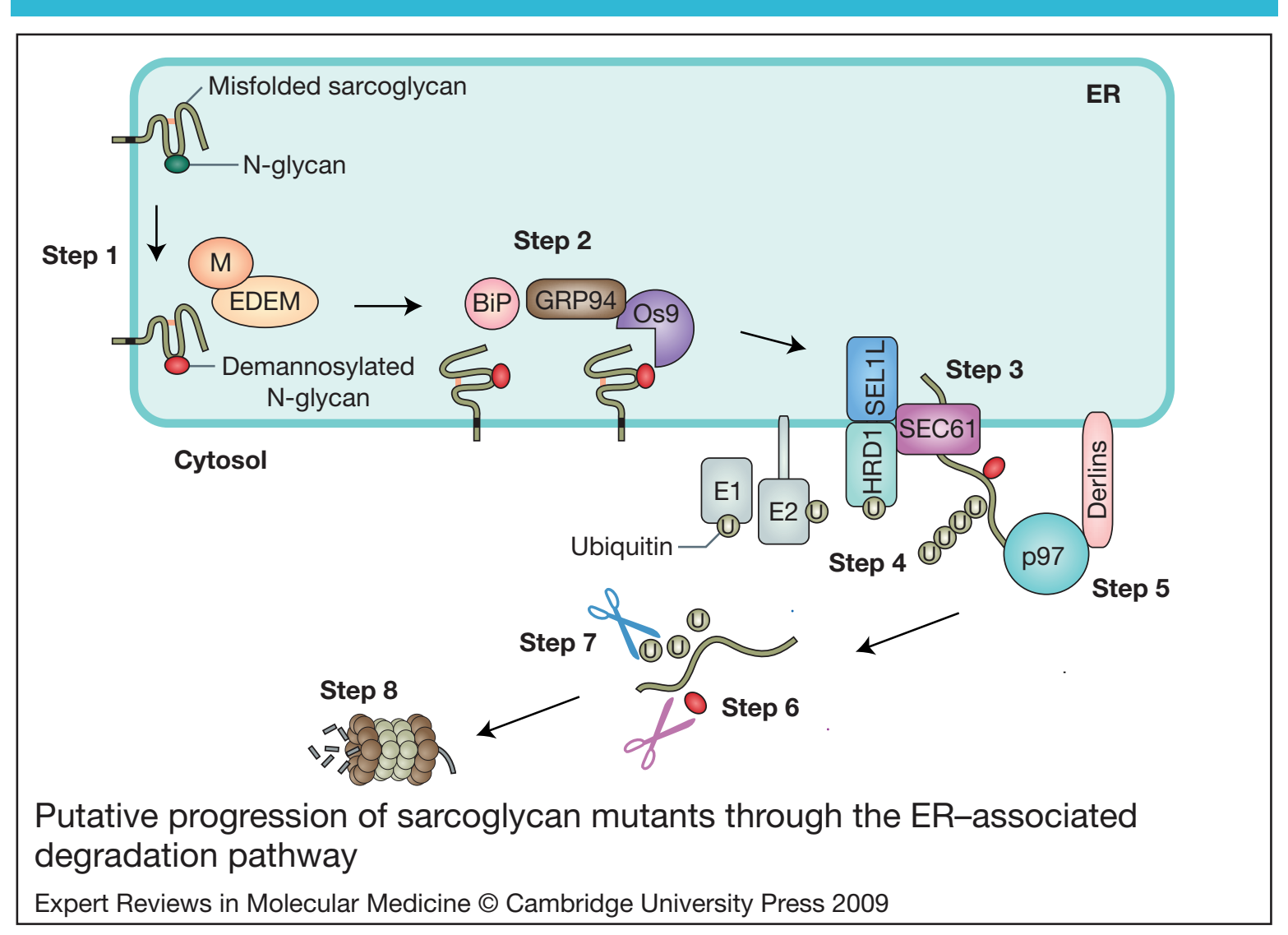

Figure 4. Putative progression of sarcoglycan mutants through the ER-associated degradation pathway. The putative processing of sarcoglycan mutants is represented in consecutive steps. Non-native sarcoglycans are targeted to ERAD by the intervention of mannosidase I and/or mannosidase-like proteins (EDEM), which remove a mannose from the $\mathrm{N}$-glycan and terminate its maturation process (Step 1). Then, the misfolded sarcoglycan is identified and escorted for retrotranslocation by a targeting complex composed of three probable components (BiP, GRP94 and Os9) (Step 2). Dislocation of the ERAD substrate occurs through the SEL1L-HRD1 complex (Step 3), which also includes Sec61, the actual channel. During retrotranslocation, the polypeptide is ubiquitinated by cytosolic and membrane-associated E1, E2 and E3 enzymes (Step 4). Note that HRD1 is one such E3 ligase. A multimeric protein complex, which comprises the p97 ATPase and Derlin proteins (through which the complex attaches to the ER membrane), dislocates the terminally misfolded sarcoglycan from the ER (Step 5). The same p97 complex targets the polyubiquitinated $(\mathrm{U})$ sarcoglycan to the $26 \mathrm{~S}$ proteasome. Approaching the 19S cap of the proteasome, the $\mathrm{N}$-glycan and the ubiquitin chain are removed by N-glycanase (Step 6) and deubiquitinating enzymes (Step 7). The polypeptide is eventually threaded into the $20 \mathrm{~S}$ proteasome catalytic core where it is broken down into small fragments (Step 8).

proteasomal inhibition by MG132 or bortezomib (Velcade) was able to rescue expression of D97G, $\mathrm{R} 98 \mathrm{H}, \mathrm{P} 228 \mathrm{Q}$ and V247M, but not R77C, $\alpha$-sarcoglycan mutants, and more importantly, the mutants formed a stable sarcoglycan complex that localised at the cell membrane (Ref. 14). This finding demonstrates that inhibition of the proteasome, the last step in the ERAD pathway (Fig. 4, Step 8), has a retrograde effect on mutant processing, permitting the assembly of partly misfolded proteins with the other sarcoglycan components, which favours their transport to the cell membrane. Moreover, these data suggest that D97G, R98H, P228Q and V247M $\alpha$-sarcoglycan mutants maintain the ability to form a potentially functional complex at the cell membrane. Regarding the inability to recover the R77C mutation, it is worth mentioning that the defective protein is known to form aggregates and is retained in the ER when expressed in heterologous cells (Refs 13, 14, 88); however, R77C $\alpha$-sarcoglycan can 
occasionally be detected at the cell membrane, either with or without proteasome inhibitor treatment (Ref. 14), suggesting that the R77C mutant might escape the ERAD system. Importantly, to prevent degradation of sarcoglycan mutants, it has been shown that it is also possible to intervene upstream of the proteasome. Mannosidase I is a key ER enzyme involved in retrotranslocation of terminally misfolded proteins (Fig. 4, Step 1). Inhibition of $\alpha$-mannosidase I, with the selective inhibitors kifunensine and deoxymannojirimycine, favoured the localisation of the R77C $\alpha$ sarcoglycan mutant at the cell membrane in HER911 cells cotransfected with $\beta-, \gamma-$ and $\delta$ sarcoglycan (Ref. 13). However, in mouse, the substitution of H77 with a cysteine does not lead to a pathological phenotype (Refs 13, 89), suggesting that if there are no differences in the protein quality control system among species, the C77 variant has noeffect on $\alpha$-sarcoglycan function.

Recent evidence indicates that pharmacological therapies could assist ER protein processing of disease-causing mutants (Ref. 90). Molecules that interfere with the ER quality control system, ERAD processing and ubiquitin proteasome degradation may result in a greater level of mutated sarcoglycan proteins, which increases their chance of reaching the cell membrane. An essential prerequisite of this salvage approach is that the rescued protein mutant maintains residual activity and/or function at the cell membrane. It is thus essential to first define the criteria for the identification of treatable mutants. Diverse computational tools, based on both protein structural and evolutionary information, are currently available to facilitate the prediction of the functional consequences of missense mutations. In general, these methods give clues about whether replaced residues are associated with a disease or are simply neutral polymorphisms. Adverse mutations can either destabilise the structure or disrupt a functional site, such as ligand binding, catalytic or proteinprotein interaction sites.

Unfortunately, structural data on the sarcoglycan complex are incomplete, so predictive approaches have to compensate for the lack of both experimental data and identification of homologous structures. In almost all cases of sarcoglycanopathy analysed so far, the mutants may be considered to be processing mutants.
However, several mutants might retain their function if they could escape the quality control system. Mutations of critical residues within hypothetical functional domains and in highly conserved sequences may be expected to have loss-of-function consequences. By contrast, substitutions in noncritical regions might generate functional proteins. Below, we provide a concise summary of mutations in the four sarcoglycans.

\section{Mutations in $\alpha$-sarcoglycan}

Seventy-three mutations have been reported in the $\alpha$-sarcoglycan gene (SGCA) that cause changes in the protein. Fifty-two are missense mutations that generate a complete protein with a single residue substitution (Fig. 5), whereas 21 mutations (nucleotide substitution, duplication, deletion or insertions) produce truncated, incomplete or anomalous proteins. All but one of the $\alpha$-sarcoglycan missense mutations are mapped in the extracellular domain - a critical region for the organisation of a stable sarcoglycan tetramer and association with dystroglycan (Refs 22, 23).

One third of the $\alpha$-sarcoglycan missense mutations map to the cadherin-like domain, which is believed to be important for $\mathrm{Ca}^{2+}$ dependent association with the similar domain present in $\alpha$-dystroglycan (Ref. 76). R34C, R34H, D97G, R98S, R98C and R98H missense mutations map to putative $\mathrm{Ca}^{2+}$-binding sites in the cadherin-like domain (Ref. 76). Substitution in each of these three critical residues (R34, D97 and R98) causes LGMD in homozygous patients (Refs 44,46). R74 causes a severe phenotype in a homozygous patient (Ref. 54). Intriguingly, substitution of D97 and R98 residues seems to produce processing mutants, because they can be pharmacologically rescued at the cell membrane (Ref. 14). The R77C missense substitution in the cadherin-like domain is the most frequently reported $\alpha$-sarcoglycan mutation. Analysis of muscle samples from homozygous R77C LGMD patients shows that $\alpha$-sarcoglycan is absent in the majority of cases, with variable residual expression of the sole $\gamma$-sarcoglycan (Refs 9, 44, 46, 47). Protein expression data, however, do not tell us whether mutations within the cadherin-like domain also compromise $\alpha$-sarcoglycan function.

It is worth noting that seven $\alpha$-sarcoglycan missense mutations cause the substitution of 
$\alpha$-sarcoglycan

1 MAETLFWTPL LVVLLAGLGD TEAQQTTLHP LVGRVFVHTL DHETFLSLPE HVAVPPAVHI

61 TYHAHLQGHP DLPRWLRYTQ RSPHHPGFLY GSATPEDRGL QVIEVTAYNR DSFDTTRQRL

121 VLEIGDPEGP LLPYQAEFLV RSHDAEEVLP STPASRELSA LGGLWEPGEL QLLNVTSALD

181 RGGRVPLPIE GRKEGVYIKV GSASPFSTCL KMVASPDSHA RCAQGQPPLL SCYDTLAPHF

241 RVDWCNVTLV DKSVPEPADE VPTPGDGILE HDPFFCPPTE APDRDFLVDA LVTLLVPLLV

301 ALLLTLLLAY VMCCRREGRL KRDLATSDIQ MVHHCTIHGN TEELRQMAAS REVPRPLSTL

361 PMFNVHTGER LPPRVDSAQV PLILDQH

$\beta$-sarcoglycan

1 MAAAAAAAAE QQSSNGPVKK SMREKAVERR SVNKEHNSNF KAGYIPIDED RLHKTGLRGR

61 KGNLAICVII LLFILAVINL IITLVIWAVI RIGPNGCDSM EFHESGLLRF KQVSDMGVIH

121 PLYKSTVGGR RNENLVITGN NQPIVFQQGT TKLSVENNKT SITSDIGMQF FDPRTQNILF

181 STDYETHEFH LPSGVKSLNV QKASTERITS NATSDLNIKV DGRAIVRGNE GVFIMGKTIE

241 FHMGGNMELK AENSIILNGS VMVSTTRLPS SSSGDQLGSG DWVRYKLCMC ADGTLFKVQV

301 TSQNMGCQIS DNPCGNTH

$\gamma$-sarcoglycan

1 MVREQYTTAT EGICIERPEN QYVYKIGIYG WRKRCLYLFV LLLLIILVVN LALTIWILKV

61 MWESPAGMGH LCVTKDGLRL EGESEFLFPL YAKEIHSRVD SSLLLQSTQN VTVNARNSEG

121 EVTGRLKVGP KMVEVQNQQF QINSNDGKPL FTVDEKEVVV GTDKLRVTGP EGALFEHSVE

181 TPLVRADPFQ DLRLESPTRS LSMDAPRGVH IQAHAGKIEA LSQMDILFHS SDGMLVLDAE

241 TVCLPKLVQG TWGPSGSSQS LYEICVCPDG KLYLSVAGVS TTCQEHSHIC L

$\delta$-sarcoglycan

1 MMPQEQYTHH RSTMPGSVGP QVYKVGIYGW RKRCLYFFVL LLMILILVNL AMTIWILKVM

61 NFTIDGMGNL RITEKGLKLE GDSEFLQPLY AKEIQSRPGN ALYFKSARNV TVNILNDQTK

121 VLTQLITGPK AVEAYGKKFE VKTVSGKLLF SADNNEVVVG AERLRVLGAE GTVFPKSIET

181 PNVRADPFKE LRLESPTRSL VMEAPKGVEI NAEAGNMEAT CRTELRLESK DGEIKLDAAK

241 IRLPRLPHGS YTPTGTRQKV FEICVCANGR LFLSQAGAGS TCQINTSVCL

\section{Sarcoglycan missense mutations}

\section{Expert Reviews in Molecular Medicine @ Cambridge University Press 2009}

Figure 5. Sarcoglycan missense mutations. Sequence of $\alpha-, \beta-, \gamma$ and $\delta$-sarcoglycan with all missense substitutions responsible for LGMD indicated in red. The indicated missense mutations are derived from the Leiden database (http://www.lovd.org) and recent reports (Refs 53, 92). The stretch of residues of the transmembrane domain is shown in yellow, the putative $\mathrm{N}$-linked glycosylation sites in green, and the predicted phosphorylation sites (Ref. 95) in dark green. In $\alpha$-sarcoglycan, the signal sequence is indicated in italics, the cadherin-like domain (Ref. 76) in grey, whereas the putative ATP-binding site (Ref. 38) is shown in blue. In $\beta-, \gamma$ - and $\delta$-sarcoglycan, the putative EGF-like sequence (Ref. 3 ) is underlined.

diverse residues with a cysteine, which are expected to cause abnormal disulphide bonds and abnormal conformation of the nascent protein. However, it has been demonstrated in a heterologous cell system that it is the replacement of a positive residue at position 77 , rather than the introduction of a cysteine, that causes retention of the mutant protein (Ref. 12). This finding highlights just how complicated the prediction of pathological consequences associated with single mutations is in proteins of undetermined function.

Two mutations, V175A and V247M, map within the $\mathrm{N}$-glycosylation sequence of $\alpha$-sarcoglycan
(174NVT and 246NVT become, respectively, 174NAT and 246NMT), which are conservative substitutions that usually do not affect glycosylation. Nevertheless, homozygous V247M causes LGMD (Refs 46, 48), suggesting that they are processing mutants. Interestingly, five additional $\alpha$-sarcoglycan missense mutations reside in the highly conserved putative $\alpha$-sarcoglycan ATP-binding domain (Ref. 38, blue box in Fig. 5). One of these, $\mathrm{R} 221 \mathrm{H}$, targets a critical residue in the ATP-binding pocket of the protein and causes a severe reduction of $\alpha$-sarcoglycan, with a slight reduction of the other

Accession information: doi:10.1017/S1462399409001203; Vol. 11; e28; September 2009 (c) Cambridge University Press 2009. Re-use permitted under a Creative Commons Licence-by-nc-sa. 
subunits (Ref. 96). Thus, R221H probably causes a loss of function, whereas substitutions in the remaining four residues did not affect the ATP-binding capacity. Nonetheless, the C232S mutation causes a severe LGMD in homozygous patients (Ref. 53).

A single $\alpha$-sarcoglycan mutation, M312R, maps in the intracellular domain, apparently without having adverse consequences on the extracellular domain; nevertheless, this mutation causes LGMD in a homozygous patient (Ref. 97). Similarly, two mutations caused by deletions generate two $\alpha$-sarcoglycan proteins with normal extracellular domains but with truncated, scrambled intracellular tails. At present, no data regarding the function of the $\alpha$-sarcoglycan intracellular tail are available. It is therefore not clear whether these mutations generate a misfolded protein intercepted by the ERAD-C machinery or whether they also abrogate unknown functions located therein.

\section{Mutations in $\beta$-sarcoglycan}

Forty-nine mutations have been so far described in the $\beta$-sarcoglycan gene (SGCB), 29 of which are missense mutations that generate a full protein with a single residue substitution (Fig. 5). Removal of glycosylation sites in $\beta$-sarcoglycan is known to affect the assembly of the complex (Ref. 85). The $\beta \delta$-sarcoglycan core is suggested to link dystrophin through undefined portions of their intracellular tail (Ref. 29); five missense mutations reside in the $\beta$-sarcoglycan tail and might have either lossof-function consequences or simply generate processing mutants; two of these (Q11G and G56R) cause LGMD in homozygous patients. In the large extracellular domain, the portion of $\beta$-sarcoglycan proximal to the transmembrane domain is thought to be required for the binding to $\delta$-sarcoglycan (Ref. 29); nine missense mutations reside in this portion of $\beta$-sarcoglycan and may thus perturb the assembly of the sarcoglycan complex. Of these nine mutations, seven cause LGMD in homozygous patients (http://www.lovd.org). Two additional missense mutations reside in apparently noncritical residues of the $\beta$-sarcoglycan cysteine-rich domain (N304S and D311N), which is a putative EGF-like sequence, and might not be functionally relevant, even though homozygous N304S causes a mild phenotype.

\section{Mutations in $\gamma$-sarcoglycan}

Forty mutations are described in the $\gamma$-sarcoglycan gene (SGCG), 16 missense mutations generating a full protein with a single residue substitution (Fig. 5) and 24 that generate a truncated protein or no protein at all. Three portions of the $\gamma$ sarcoglycan extracellular domain display possible critical functions, two for the assembly with either $\beta$-sarcoglycan or $\alpha$-sarcoglycan, and the putative EGF-like domain. Residues 60-155 in the $\gamma$-sarcoglycan portion proximal to the transmembrane domain are thought to be essential for the interaction with $\alpha$ - and $\beta$ sarcoglycan (Ref. 29). Although the relevant residues for binding to the sarcoglycan partners are not known, it is expected that some of the missense mutations residing in this region compromise the correct assembly of the complex. The homozygous Q82R mutation actually causes severe LGMD and the almost complete absence of the four sarcoglycans from the cell membrane (Ref. 53). However, again, some of these might simply be processing mutants. In $\mathrm{CHO}$ cells, mutations producing a truncated $\gamma$-sarcoglycan without the cysteines in the EGF-like domain do not permit sarcoglycan complex assembly (Ref. 82), as demonstrated in muscle samples from patients (Ref. 98). By contrast, in two LGMD-2C patients, a homozygous $\Delta 525 \mathrm{~T}$ mutation generates a truncated $\gamma$-sarcoglycan protein without the EGF-like domain, which is apparently able to assemble with the other sarcoglycans (Ref. 45). The C283Y missense mutation in the $\gamma$-sarcoglycan cysteine-rich domain could be functionally relevant, because this cysteine is crucial in the EGF-like domain (Ref. 98). In fact, C283Y can cause severe LGMD and it severely perturbs the assembly of $\alpha$-sarcoglycan to the complex in COS-1 cells (Ref. 85). Intriguingly, the L53P mutation in the transmembrane domain causes mild LGMD with the complete absence of the protein but traces of the other sarcoglycans (Ref. 53).

\section{Mutations in $\delta$-sarcoglycan}

Fourteen $\delta$-sarcoglycan gene $(S G C D)$ mutations have been so far found to cause LGMD-2F. Eight are missense substitution and all reside in the extracellular portion (Fig. 5). The portion in the extracellular domain between residues 57 and 92 of $\delta$-sarcoglycan (proximal to the transmembrane domain) was shown to be critical for the association with $\beta$-sarcoglycan

Accession information: doi:10.1017/S1462399409001203; Vol. 11; e28; September 2009 (c) Cambridge University Press 2009. Re-use permitted under a Creative Commons Licence-by-nc-sa. 
(Ref. 29). Three disease-causing mutations are within this portion. Considering the critical role of the $\beta \delta$-sarcoglycan core in the assembly of sarcoglycans, these substitutions are expected to have functional consequences. Similarly, the portion between residues 92 and 200 is believed to be important for $\delta$ - to $\gamma$-sarcoglycan interaction (Ref. 29). One substitution, R198P, resides in a recently identified highly conserved sequence (L190RLESPTRSL) (Ref. 29). As highly conserved sequences are predicted to be functionally important, it is not surprising that homozygous expression causes a DMD-like phenotype (Refs 7, 48). Five $\delta$-sarcoglycan mutations generate a protein with a truncated extracellular domain and cause clinical phenotypes from LGMD to DMD-like in homozygous patients.

To date, there is not sufficient information to precisely predict the number of mutations in the four sarcoglycans that cause loss-of-function consequences or which result in processing mutants. We estimate that about half of LGMDcausing missense mutations in the four sarcoglycans are potentially recoverable to the cell membrane, by preventing their premature degradation through pharmacological interference with the ERAD machinery. This percentage could be even higher if the residues substituted within supposedly functional sarcoglycan regions do not affect that function. To this end, studies in heterologous cell systems could facilitate the identification of critical residues of sarcoglycan functional domains. Heterologous cells could be used in preliminary tests for the identification of appropriate molecules and/or interventions that would favour the recovery of mutant sarcoglycans.

\section{Therapeutic approaches for sarcoglycanopathies}

Sarcoglycanopathies are autosomal recessive disorders. The majority of LGMD cases are due to compound heterozygous conditions and only a small percentage result from homozygous mutations. This situation could be advantageous to some extent, because even partial restoration of a functional protein might correct the dystrophic phenotype. Two therapeutic approaches are currently being investigated for delivery of healthy genes into dystrophic muscles: gene and cell therapy (Refs 99, 100, 101). Gene therapy has been carried out in all animal models of sarcoglycanopathies, and is aided by the small size of the cDNA. The most effective gene delivery was obtained with nonpathogenic adeno-associated viruses (reviewed in Ref. 100). Importantly, in animal models of sarcoglycanopathies, the best results were seen when the vector was injected before the onset of the pathology (Refs 102, 103, 104). However, to be effective in patients, gene therapy must overcome critical issues, such as targeting the largest tissue of the body and low distribution of the vector, as well as the immune response (Ref. 101).

Grafting of genetically modified or healthy donor cells is another approach to treat dystrophic muscle. Several stem cell populations exhibit myogenic potential and have been analysed for their ability to correct the dystrophic phenotype. These include muscle-derived satellite cells, bonemarrow-derived mesenchymal stem cells, muscle side-population cells, and cells derived from blood vessel walls, such as mesangioblasts or pericytes (reviewed by Refs 99, 105). Although a pluripotent bone-marrow-derived side population of stem cells failed to deliver $\delta$ sarcoglycan (Ref. 106), muscle satellite cells have been more successful (Ref. 107). Mesangioblasts injected into muscles of $\alpha$-sarcoglycan-null mice and dystrophin-deficient dogs show potential for gene delivery (Refs 108, 109). In addition to the difficulty of treating the most abundant tissue of the body, stem cell therapy presents several potential disadvantages, such as the requirement for immunosuppression, difficulty in identification of suitable adult stem cells, and possible diffusion and colonisation efficiencies. Last, but not least, caution must be used in transferring the encouraging results obtained in animal models to humans.

Pharmacological strategies are becoming an important alternative to gene delivery, because drugs can more easily target each tissue of the body. In general, pharmacological therapies can be directed either to circumvent the primary defect or to treat its pathological consequences. Recent evidence indicates that this approach can also be used to facilitate the processing of misfolded but functional protein mutants. The first treatment to produce beneficial effects was the use of corticosteroids in DMD; however, besides the anti-inflammatory effect, the mechanisms of action remain undefined. Another pharmaceutical approach successfully overcame premature stop codons in mutated mRNAs, thus

Accession information: doi:10.1017/S1462399409001203; Vol. 11; e28; September 2009 (c) Cambridge University Press 2009. Re-use permitted under a Creative Commons Licence-by-nc-sa. 
favouring translational read-through (Refs 101, 110). Initially, this approach used aminoglycoside antibiotics, which successfully rescued dystrophin in $m d x$ mice (Ref. 111) and was the object of clinical trials in DMD patients. However, additional work in $m d x$ mice demonstrated divergent results (Ref. 112). Recently, PTC124, a very promising nonaminoglycoside agent, was demonstrated to selectively induce ribosomal read-through of premature stop codons but not the normal stop codon (Refs 113, 114). The use of specific antisense oligonucleotides to induce exon skipping and generate in-frame transcripts translated into functional, albeit smaller, protein could be an additional approach for the therapy of diseases caused by mutations generating premature stop codons. This approach is useful for large proteins possessing many repeated functional units (e.g. dystrophin spectrin-like repeats), some of which can be deleted without dramatic functional consequences (Refs 101, 115). This option is not applicable to sarcoglycanopathies because there are no dispensable domains in the extracellular portion of sarcoglycans and the genomic structure of the sarcoglycan genes prevents any meaningful exon skipping.

Alternative strategies aim to compensate for the muscle mass wasting or to directly cope with the pathological mechanisms in muscular dystrophy (reviewed by Ref. 100). However, contrasting results were obtained by targeting the activity of regulators of muscle growth. For example, treatment with insulin-like growth factor-1 (IGF-1) improved life expectancy of the $\delta$-sarcoglycandeficient hamster (Ref. 116) and beneficial effects were obtained by treating $\alpha$-sarcoglycan-deficient mice with a deacetylase inhibitor that induced the expression of the myostatin inhibitor follistatin in satellite cells (Ref. 117). By contrast, administration of inactive myostatin ameliorated the dystrophic phenotype of calpain 3 but not of $\alpha$-sarcoglycan-null mice (Ref. 118). Similarly, the antibody-mediated inhibition of myostatin in $\gamma$-sarcoglycan-deficient mice was ineffective (Ref. 119). Additional work is thus necessary to explore this important therapeutic option for sarcoglycanopathies.

\section{Possible pharmacological treatment of sarcoglycan-processing mutants}

As indicated above, all the sarcoglycan mutants produced by single-residue substitutions can be considered to be processing mutants. Therefore the possibility to intervene in the different steps of the cell quality control system offers great opportunities for therapeutic intervention. Considering that the majority of LGMD patients are compound heterozygotes, it is reasonable to expect that rescue of just one of the two allele products would ameliorate the dystrophic phenotype.

Chemical and pharmacological chaperones can assist processing mutants by stabilising the defective protein. Chemical chaperones create a more favourable environment for protein mutant processing and trafficking. Typical chemical chaperones are small molecules (e.g. glycerol, dimethyl sulfoxide, 4-phenylbutyrate, trimethylamine) that stabilise the folding process of mutant proteins and/or the assembly of multimeric complexes by increasing hydration of the folding mutant (Ref. 90). However, to be effective, these molecules must be used at very high concentrations, a condition that rarely permits their utilisation in clinical trials.

Numerous molecules have been proposed as pharmacological chaperones, which might be more useful than chemical chaperones because they act in a more specific manner, are less toxic and are utilised at lower levels. An ideal pharmacological chaperone is a molecule that interacts with the functional sites of the mutant, stabilising the protein until it reaches the cell membrane. For example, ligands of cell membrane receptors have been shown to stabilise and facilitate their processing (reviewed by Ref. 90). $\beta-, \gamma$ - and $\delta$-sarcoglycan possess an EGF-like domain in their extracellular portion (Ref. 3), although the putative ligand modulating this receptor-like function is unknown. Identification of this factor would reveal a possible crucial function of the sarcoglycan complex and provide a candidate drug to promote the processing of sarcoglycan complex.

As illustrated above, there are additional sites for intervention to promote the processing of potentially functional sarcoglycan mutants. In addition to a general intervention aimed at modification of the ER environment towards more favourable conditions (produced by molecular chaperones that affect oxidative state or calcium levels), other more specific actions can be envisaged. The eight steps indicated in

Accession information: doi:10.1017/S1462399409001203; Vol. 11; e28; September 2009 (c) Cambridge University Press 2009. Re-use permitted under a Creative Commons Licence-by-nc-sa. 
Figure 4 are all suitable targets for pharmaceutical intervention. The retrograde beneficial effects produced by inhibiting the final steps of the proteasome degradation process (Step 8) suggest that intervention in the intermediate steps of the ERAD pathway might also favour a reduction of sarcoglycan mutant disposal, permitting their assembly into a complete tetramer, and trafficking to the cell membrane. Below, we provide a concise description of the ERAD-ubiquitinproteasome pathway.

The ERAD process can be split into three basic parts: (1) initial recognition of the ERAD substrate; (2) targeting and retrotranslocation of the ERAD substrate from the ER into the cytosol; and (3) ubiquitination and degradation of the ERAD substrate into small peptides within the 20S proteasome core (Fig. 4) (Refs 11, 12, 120). Sarcoglycans mature in the ER membrane, and are processed by distinct pathways of the quality control machinery, according to whether the defects reside in cytosolic, transmembrane or extracellular (lumenal) domains (Refs 11, 120, 121). Three distinct ERAD pathways are in fact used according to the topology of the misfolded protein. ER-soluble lumenal proteins and integral membrane proteins with misfolded extracellular (lumenal) domains are degraded via the ERAD-L pathway, membrane proteins with cytosolic defects are degraded by the ERAD-C pathway, whereas those with misfolded transmembrane domains are degraded by the ERAD-M pathway. As most of sarcoglycans mutations reside in the extracellular domain, ERAD-L is almost certainly used for their degradation. Since some patients have defects in the intracellular portion of $\alpha$ - and $\beta$-sarcoglycan, cytosolic ER-associated components of the ERAD-C pathway could instead be involved in processing of these mutants (Ref. 120).

Exit of misfolded proteins from the calnexincalreticulin cycle is determined by the 'inactivity' of UDP-glucose:glycoprotein glucosyltransferase1 (UGGT) (Fig. 3). Mannosidase I is a key enzyme in directing misfolded proteins to ERAD; by trimming terminal mannoses, mannosidase I inhibits glucose readdition and thus re-entry into the calnexin-calreticulin cycle. In this initial recognition function, mannosidase I is assisted by a family of mannosidase-like proteins, EDEM1-EDEM3, which operate both as mannosidases and chaperones (Fig. 4, Step 1)
(Ref. 12). Before directing terminally misfolded protein to retrotranslocation to the cytosol, ERAD substrates are reduced by protein disulfide isomerases (PDIs), such as the oxidoreductase ERp57. This action probably requires the assistance of BiP (GRP78). The ER surveillance apparatus can also detect redundant monomers of unassembled multiprotein complexes, which may be the case for sarcoglycans, causing the degradation of correctly folded proteins, whose recognition as ERAD substrates remains an intriguing process.

Subsequently, a composite lumenal and/or membrane-associated surveillance system targets ERAD substrates for retrotranslocation and degradation. Sarcoglycans defective in the extracellular domain, which represent the vast majority of cases, are probably recognised by the ER lectins OS-9 and XTP3-B and the chaperones BiP and GRP94 (Fig. 4, Step 2) (Ref. 122), and then recruited to a transmembrane complex that operates the retrotranslocation process. This complex is formed by SEL1 and HRD1, with SEL1 facing the lumenal side and HRD1, an E3 ubiquitin ligase, the cytosolic ER side (Ref. 120) (Fig. 4, Step 3). The presence of a ubiquitin ligase (HRD1) in this complex indicates that ubiquitination is strictly associated with translocation of ERAD substrates (Ref. 120). Ubiquitination of proteins is in fact a prerequisite for their degradation. This process is operated by the concerted and sequential action of three families of enzymes: E1 and E2 enzymes that respectively activate and conjugate ubiquitin to E3 enzymes, which eventually ligate ubiquitin to lysine-competent residues in the ERAD substrate (Fig. 4, Step 4). Sec61, which controls cotranslational entry of nascent proteins, is probably the channel protein through which luminal misfolded proteins pass from the ER to the cytosol. In addition, Derlin proteins have been found in the retrotranslocation machinery, and possibly form a pore themselves (Ref. 123).

A cytosolic complex comprising the ATPase p97, UFD1 and NPL4 is at the crossroads of all three ERAD pathways, with p97 providing the energy for retrotranslocation of monoubiquitinated ERAD substrates (Refs 120, 124). The p97-UFD1-NPL4 complex, associated with the ER membrane via Derlin proteins (Fig. 4, Step 5), escorts the polyubiquitinated mutant protein 
destined to degradation toward the proteasome (Ref. 120). Next, the polyubiquitinated protein is deglycosylated by the cytosolic enzyme peptide:N-glycanase (PNGase) (Fig. 4, Step 6), deubiquitinated (Fig. 4, Step 7) and finally degraded in the $20 \mathrm{~S}$ proteasome core (Ref. 125) (Fig. 4, Step 8).

It is important to mention that accumulation of misfolded proteins in the ER lumen may elicit the unfolded protein response (UPR) signal transduction pathway (Ref. 126). The function of the UPR is to quickly remove accumulated aberrant substrates, which are frequently in the form of aggregates, and restore the ER to an efficiently operating maturation compartment. Activation of the UPR causes (1) upregulation of ER-resident protein expression to facilitate the proper folding of misfolded proteins; (2) reduced activity of the secretory pathway by decreasing the expression of secretory cargo; (3) increased degradation of terminally misfolded proteins; and (4) restrained translational initiation until the abnormality has been minimised. However, long-lasting activation of the UPR might have severe consequences for cell survival (Ref. 126). Therefore, prolonged interference with the ERAD pathway should be considered cautiously.

In summary, we propose that misfolded or truncated sarcoglycans are intercepted by the ER quality control system, which sequentially activates ERAD, ubiquitin-proteasome degradation and possibly UPR, to rapidly remove defective proteins and to maintain an efficient ER. Table 2 lists potential molecular targets for therapeutical intervention within the diverse components of the ERAD machinery that could promote the rescue of misfolded sarcoglycan mutants. To this end, it would also be important to determine whether the sarcoglycan complex is specifically targeted in ERAD degradation; the identification of musclespecific quality control or ERAD factors that participate in the degradation process of sarcoglycan mutants would be of a great help in the development of novel therapeutical approaches. For example, although glycoproteins are bound to calnexin and/or calreticulin, disulphide bond formation and folding are promoted by the recruitment of ERp57, a member of the protein disulphide isomerase family. ERp57 is essential for efficient folding of glycoproteins sharing common structural domains, for instance Type 1 transmembrane proteins, such as $\alpha$-sarcoglycan, and proteins that possess EGF-like domains, such as $\beta-, \gamma$ - and $\delta$-sarcoglycan (Ref. 127). ERp57 or an analogous ER component could thus be involved in the processing of sarcoglycans and would be a possible target for therapy. Interestingly, some antibiotics have been recently described to inhibit oxidoreductase activity of ERp57 (Ref. 128).

Biochemical and gene-silencing approaches have identified many of the ERAD components indicated in Fig. 4, but have also demonstrated that modification of their activity might have substantial consequences on the fate of processing mutants. The first and most widely studied ERAD substrate is the cystic fibrosis transmembrane regulator (CFTR), a cell membrane chloride channel whose mutations are responsible for cystic fibrosis (Refs 90, 129, 130). $\Delta$ F508 is the most frequent mutation of CFTR that causes misfolding of a partially functional protein. Similarly to $\alpha$-sarcoglycan mutants, proteasome inhibition (Fig. 4, Step 8) permitted the partial rescue of $\Delta$ F508 CFTR (Refs 131, 132). Miglustat (N-butyldeoxynojirimycin), an inhibitor of $\alpha-1,2$ glucosidase (Fig. 3b), effectively corrected the $\Delta$ F508 and G622D CFTR mutants in nasal epithelial cells through an action that interferes with the progress of the misfolded protein in the calnexin-calreticulin cycle (Ref. 131). Geldanamycin treatment in vitro causes the release of CFTR protein from competent chaperones and the concomitant termination of ubiquitination and formation of a stable protein (Ref. 133). Derlin-1 (Fig. 4, Step 5) is thought to recognise misfolded nonubiquitinated CFTR, and reduction of its expression led to significantly increased levels of $\Delta$ F508 CFTR (Ref. 134). The p97 ATPase (Fig. 4, Step 5) is also a potential target for pharmacological therapy of sarcoglycanopathies, because downregulation of p97 partly rescued $\Delta$ F508 CFTR (Ref. 132). However, a similar approach must be considered very cautiously in skeletal muscle, because mutations affecting p97 activity are responsible for inclusion body myopathy, which is characterised by excessive accumulation of ubiquitinated proteins (Ref. 135). Conversely, a novel inhibitor of p97, Eeyarestatin I, was shown to efficiently block degradation of ERAD substrates (Ref. 136). N-glycanase is the sole deglycosylating enzyme directing misfolded proteins to $26 \mathrm{~S}$ proteasome (Fig. 4, Step 6). 
Table 2. ER quality control and ERAD targets for possible therapy

\section{Location}

ER quality control

Lumenal and membraneassociated ERAD recognition and targeting

ERAD membrane-associated dislocating proteins

ERAD membrane associated and cytosolic ubiquitin enzymes

ER cytosol and membrane associated

Proteasome

\begin{tabular}{|c|c|}
\hline Target & Activity or func \\
\hline PDI & Disulphide isomera \\
\hline ERp57 & Oxidoreductase, is \\
\hline UGGT & Folding sensor \\
\hline Mannosidase I & $\begin{array}{l}\text { Removal of manno } \\
\text { protein N-glycans }\end{array}$ \\
\hline EDEM1-3 & Mannosidase-like \\
\hline GRP94 & $\begin{array}{l}\text { Chaperone, folding } \\
\text { assistant }\end{array}$ \\
\hline $\mathrm{BiP}$ & $\begin{array}{l}\text { Chaperone, folding } \\
\text { assistant }\end{array}$ \\
\hline OS9 & $\begin{array}{l}\text { Lectin, substrate } \\
\text { recognition }\end{array}$ \\
\hline XTP3-B & $\begin{array}{l}\text { Lectin, substrate } \\
\text { recognition }\end{array}$ \\
\hline
\end{tabular}

SEL1L

HRD1

Sec61p

Derlins

E1

E2

E3, HRD1

E4

p97-UFD1NPL4

p97

Derlins

$\mathrm{N}$-glycanase

DUB

20S

proteasome
ER component associated with HRD1

Transmembrane E3

Ubiquitin ligase

Possible retrotranslocon

channel

Component of

retrotranslocon channel

E1 ubiquitin-activating

enzymes

E2 ubiquitin-conjugating

enzymes

E3 ubiquitin ligases

Polyubiquitin enzymes

ERAD protein extraction complex

ATPase providing energy for substrate extraction

ER anchor for p97-UFD1NPL4 complex

Removal of N-glycans before degradation

Removal of ubiquitin before degradation

Degradation of ERAD substrate $\quad$ Drug
Ribostamycin
Vancomycin
Kifunensine an
deoxyMR

(a) 
Inhibition of N-glycanase, by very low doses of the pan-caspase inhibitor z-VAD-fmk, or by the novel inhibitor chloroacetamidyl chitobiose (Ref. 137), was shown to prevent deglycosylation and slow down retrotranslocation of misfolded proteins (Ref. 138). This section and Table 2 briefly summarise some of the diverse approaches used for the treatment of CFTR processing mutants that could also be applied to sarcoglycans.

Finally, it is important to note that proteasome inhibition has been successfully used to rescue the expression and localisation of components of the DGC in skeletal muscle of $m d x$ mice, and DMD and BDM explants, an effect obtained by reducing the elevated dismantling rate of disassembled proteins, which is due to the absence of dystrophin (Refs 140, 141, 142). Recently, it has been shown that $\varepsilon$-sarcoglycan mutants that cause myoclonus-dystonia syndrome, are also intercepted by ER quality control and degraded by the ubiquitin-proteasome system (Ref. 143), suggesting that interfering with the ERAD process could be beneficial, even with this syndrome. Last, but not least, recent evidence shows that inhibition of the proteasome with the FDA-approved inhibitor bortezomib (Velcade) successfully rescued the localisation of D97G $\alpha$-sarcoglycan mutant in muscle explants from an LGMD-2D patient (Ref. 14). Additional studies are certainly necessary, but current knowledge offers an exciting perspective for the pharmacological treatment of sarcoglycan processing mutants.

\section{Clinical implications}

It is worth reporting that no clinical trials are underway for the pharmaceutical treatment of sarcoglycanopathies. However, recent research shows some promise (Refs 13,14), particularly with sarcoglycan mutations tightly linked to the ER quality control system. Evidence indicates that interfering with the ERAD machinery may be a relatively safe approach. Apparently, inhibition of mannosidase I with kifunensine does not produce pathological consequences in mice (Ref. 13). Moreover, inhibition of the proteasome with bortezomib is already in Phase III clinical trials for the treatment of multiple myeloma (http://www.velcade.com). Thus, both drugs have the potential for treating sarcoglycanopathy patients. In this review, we highlight additional components within the ERAD pathway whose activity could be regulated to favour processing of sarcoglycan mutants. Both currently used and novel drugs are emerging as suitable candidates for the pharmaceutical treatment of sarcoglycanopathies, and research should concentrate in ERADmodulating compounds with the aim of identifying promising therapeutical approaches for future clinical trials.

\section{Outstanding research questions}

Research on ERAD pathways and the proteasome system is dynamic, and novel components are continuously being discovered. Research on muscle-specific ERAD components should be encouraged and the discovery of sarcoglycanspecific chaperones and/or enzymes specifically assisting their processing would offer new avenues for future therapy. It is hoped that many molecules will be identified as potential candidates for the treatment of sarcoglycanopathies. To this end, however, preclinical studies are needed to validate their effectiveness and possible unwanted consequences. Preliminary studies in heterologous cell systems will be mandatory to test the pharmacology and toxicity of potential candidates in preparation for clinical trials.

\section{Acknowledgements and funding}

R.B. is funded by the Italian National Research Council (C.N.R.) and the Association Francaise contre les Miopathies. D.S. is funded by an Athenaeum Project of the University of Padova. The contribution of the Italian Space Agency is gratefully acknowledged. Comments from the peer reviewers are greatly appreciated.

\section{References}

1 Blake, D.J. et al. (2002) Function and genetics of dystrophin and dystrophin-related proteins in muscle. Physiological Reviews 82, 291-329

2 Davies, K.E. and Nowak, K.J. (2006) Molecular mechanisms of muscular dystrophies: old and new players. Nature Reviews. Molecular Cell Biology 7, 762-773

3 Hack, A.A. et al. (2000) Sarcoglycans in muscular dystrophy. Microscopy Research and Technique 48, 167-180

4 Ozawa, E. et al. (2005) Molecular and cell biology of the sarcoglycan complex. Muscle and Nerve 32, 563-576

5 Bönnemann, C.G. et al. (1995) $\beta$-Sarcoglycan (A3b) mutations cause autosomal recessive muscular 
dystrophy with loss of the sarcoglycan complex. Nature Genetics 11, 266-273

6 Lim, L.E. et al. (1995) $\beta$-Sarcoglycan: characterization and role in limb-girdle muscular dystrophy linked to 4q12. Nature Genetics 11, 257-265

7 Nigro, V. et al. (1996) Autosomal recessive limbgirdle muscular dystrophy, LGMD2F, is caused by a mutation in the $\delta$-sarcoglycan gene. Nature Genetics 14, 195-198

8 Noguchi, S. et al. (1995) Mutations in the dystrophin-associated protein $\gamma$-sarcoglycan in chromosome 13 muscular dystrophy. Science 270, 819-822

9 Roberds, S.L. et al. (1994) Missense mutations in the adhalin gene linked to autosomal recessive muscular dystrophy. Cell 78, 625-633

10 Angelini, C. et al. (1999) The clinical spectrum of sarcoglycanopathies. Neurology 52, 176-179

11 Anelli, T. and Sitia, R. (2008) Protein quality control in the early secretory pathway. EMBO Journal 27, 315-327

12 Hebert, D.N. and Molinari, M. (2007) In and out of the ER: Protein folding, quality control, degradation, and related human diseases. Physiological Reviews 87, 1377-1408

13 Bartoli, M. et al. (2008) Mannosidase I inhibition rescues the human $\alpha$-sarcoglycan R77C recurrent mutation. Human Molecular Genetics 17, 1214-1221

14 Gastaldello, S. et al. (2008) Inhibition of proteasome activity promotes the correct localization of disease-causing $\alpha$-sarcoglycan mutants in HEK293 cells constitutively expressing $\beta-, \gamma^{-}$, and $\delta$-sarcoglycan. American Journal of Pathology $173,170-181$

15 Ervasti, J.M. (2007) Dystrophin, its interactions with other proteins, and implications for muscular dystrophy. Biochimica et Biophysica Acta 1772, 108-117

16 Moorwood, C. (2008) Syncoilin, an intermediate filament-like protein linked to the dystrophin associated protein complex in skeletal muscle. Cellular and Molecular Life Sciences 65, 2957-2963

17 Durbeej, M., Henry, M.D. and Campbell, K.P. (1998) Dystroglycan in development and disease. Current Opinion in Cell Biology 10, 594-601

18 Ayalon, G. et al. (2008) An ankyrin-based mechanism for functional organization of dystrophin and dystroglycan. Cell 135, 1189-1200

19 Petrof, B.J. et al. (1998) Dystrophin protects the sarcolemma from stresses developed during muscle contraction. Proceedings of the National Academy of Sciences of the United States of America 90, 3710-3714

20 Helbling-Leclerc, A. et al. (1995) Mutations in the laminin $\alpha 2$ chain gene (LAMA2) cause merosindeficient congenital muscular dystrophy. Nature Genetics 11, 216-218

21 McNally, E.M. and Pytel (2007) Muscle diseases: the muscular dystrophies. Annual Review of Pathology Mechanisms of Disease 2, 87-109

22 Chan, Y.M. et al. (1998) Molecular organization of sarcoglycan complex in mouse myotubes in culture. Journal of Cell Biology 143, 2033-2044

23 Brenman, J.E. et al. (1995) Nitric oxide synthase complexed with dystrophin and absent from skeletal muscle sarcolemma in Duchenne muscular dystrophy. Cell 82, 743-752

24 Sakamoto, A. et al. (1997) Both hypertrophic and dilated cardiomyopathies are caused by mutation of the same gene, $\delta$-sarcoglycan, in hamster: an animal model of disrupted dystrophin-associated glycoprotein complex. Proceedings of the National Academy of Sciences of the United States of America 94, 13873-13878

25 Yoshida, M. et al. (2000) Biochemical evidence for association of dystrobrevin with the sarcoglycansarcospan complex as a basis for understanding sarcoglycanopathy. Human Molecular Genetics 9, 1033-1040

26 Crosbie, R.H. et al. (1997) Sarcospan, the 25-kDa transmembrane component of the dystrophinglycoprotein complex. Journal of Biological Chemistry 272, 31221-31224

27 Lebakken, C.S. et al. (2000) Sarcospan-deficient mice maintain normal muscle function. Molecular and Cellular Biology 20, 1669-1677

28 Rafii, M.S. et al. (2006) Biglycan binds to $\alpha$ - and $\gamma$ sarcoglycan and regulates their expression during development. Journal of Cellular Physiology 209, 439-447

29 Chen, J. et al. (2006) Identification of functional domains in sarcoglycans essential for their interaction and plasma membrane targeting. Experimental Cell Research 312, 1610-1625

30 Thompson, T.G. et al. (2000) Filamin 2 (FLN2): A muscle-specific sarcoglycan interacting protein. Journal of Cell Biology 148, 115-126

31 Anastasi, G. et al. (2004) Evaluation of sarcoglycans, vinculin-talin-integrin system and filamin 2 in $\alpha$ - and $\gamma$-sarcoglycanopathy: an immunohistochemical study. International Journal of Molecular Medicine 14, 989-999 
32 Assereto, S. et al. (2008) Aquaporin-4 expression is severely reduced in human sarcoglycanopathies and dysferlinopathies. Cell Cycle 7, 2199-2207

33 Lapidos, K.A. et al. (2004) The dystrophin glycoprotein complex: signaling strength and integrity for the sarcolemma. Circulation Research 94, 1023-1031

34 Yoshida, M. et al. (1998) Bidirectional signaling between sarcoglycans and the integrin adhesion system in cultured L6 myocytes. Journal of Biological Chemistry 273, 1583-1590

35 Allikian, M.J. et al. (2004) Genetic compensation for sarcoglycan loss by integrin a7b1 in muscle. Journal of Cell Science 117, 3821-3830

36 Chen, J. et al. (2007) The $16 \mathrm{kDa}$ subunit of vacuolar $\mathrm{H}^{+}$-ATPase is a novel sarcoglycan-interacting protein. Biochimica et Biophysica Acta 1772, 570-579

37 Barton, E.R. (2006) Impact of sarcoglycan complex on mechanical signal transduction in murine skeletal muscle. American Journal of Physiology 290, C411-419

38 Betto, R. et al. (1999) Ecto-ATPase activity of $\alpha$-sarcoglycan (adhalin). Journal of Biological Chemistry 19, 7907-7912

39 Sandonà, D. et al. (2004) Characterization of the ATP-hydrolyzing activity of $\alpha$-sarcoglycan. Biochemical Journal 381, 105-112

40 Sandonà, D. et al. (2005) The T-tubule membrane ATP-operated $\mathrm{P} 2 \mathrm{X} 4$ receptor influences contractility of skeletal muscle. FASEB Journal 19, 1184-1186

41 Li, D. et al. (2009) Sub-physiological sarcoglycan expression contributes to compensatory muscle protection in mdx mice. Human Molecular Genetics. 2009

42 Barresi, R. et al. (1997) Concomitant deficiency of $\beta$ - and $\gamma$-sarcoglycan in $20 \alpha$-sarcoglycan (adhalin)-deficient patients: immunohistochemical analysis and clinical aspects. Acta Neuropathologica 91, 28-35

43 Bönnemann, C.G. et al. (1996) Genomic screening for $\beta$-sarcoglycan gene mutations: missense mutations may cause severe limb-girdle muscular dystrophy type 2E (LGMD 2E). Human Molecular Genetics 5, 1953-1961

44 Carrié, A. et al. (1997) Mutational diversity and hot spots in the $\alpha$-sarcoglycan gene in autosomal recessive muscular dystrophy (LGMD2D). Journal of Medical Genetics 34, 470-475

45 Crosbie, R.H. et al. (2000) Molecular and genetic characterization of sarcospan: insights into sarcoglycan-sarcospan interactions. Human Molecular Genetics 9, 2019-2027
46 Duggan, D.J. (1997) Mutations in the sarcoglycan genes in patients with myopathy. New England Journal of Medicine 336, 618-624

47 Jung, D. et al. (1996) Characterization of $\delta$ sarcoglycan, a novel component of the oligomeric sarcoglycan complex involved in limb-girdle muscular dystrophy. Journal of Biological Chemistry 271, 32321-32329

48 Moreira, E.S. et al. (2003) Genotype-phenotype correlations in 35 Brazilian families with sarcoglycanopathies including the description of three novel mutations. Journal of Medical Genetics 40, e12

49 Piccolo, F. et al. (1995) Primary adhalinopathy: a common cause of autosomal recessive muscular dystrophy of variable severity. Nature Genetics 10, 243-245

50 Draviam, R.A. et al. (2001) Confocal analysis of dystrophin protein complex in muscular dystrophy. Muscle and Nerve 24, 262-272

51 Duclos, F. et al. (1998) Beta-sarcoglycan: genomic analysis and identification of a novel missense mutation in the LGMD2E Amish isolate.

Neuromuscular Disorders 8, 30-38

52 Fanin, M. and Angelini, C. (1999) Regeneration in sarcoglycanopathies: expression studies of sarcoglycans and other muscle proteins. Journal of the Neurological Sciences 165, 170-177

53 Klinge, L. et al. (2008) Sarcoglycanopathies: Can muscle immunoanalysis predict the genotype? Neuromuscular Disorders 18, 934-941

54 Vainzof, M. et al. (1996) The sarcoglycan complex in the six autosomal recessive limb-girdle muscular dystrophies. Human Molecular Genetics 5, 1963-1969

55 Bönnemann, C.G. et al. (2002) Primary $\gamma$-sarcoglycanopathy (LGMD 2C): broadening of the mutational spectrum guided by the immunohistochemical profile. Neuromuscular Disorders 12, 273-280

56 Higuchi, I. et al. (1998) Different manners of sarcoglycan expression in genetically proven $\alpha$-sarcoglycan deficiency and $\gamma$-sarcoglycan deficiency. Acta Neuropathologica 96, 202-206

57 Matsumura, K. et al. (2005) Proteolysis of $\beta$-dystroglycan in muscular diseases. Neuromuscular Disorders 15, 336-341

58 Duclos, F. et al. (1998) Progressive muscular dystrophy in $\alpha$-sarcoglycan-deficient mice. Journal of Cell Biology 142, 1461-1471

59 Araishi, K. et al. (1999) Loss of the sarcoglycan complex and sarcospan leads to muscular 
dystrophy in $\beta$-sarcoglycan-deficient mice. Human Molecular Genetics 8, 1589-1598

60 Coral-Vasquez, R. et al. (1999) Disruption of the sarcoglycan-sarcospan complex in vascular smooth muscle: a novel mechanism for cardiomyopathy and muscular dystrophy. Cell 98, 465-474

61 Durbeej, M. et al. (2000) Disruption of the $\beta$-sarcoglycan gene reveals pathogenetic complexity of limb-girdle muscular dystrophy type 2E. Molecular Cell 5, 141-151

62 Hack, A.A. et al. (1998) $\gamma$-Sarcoglycan deficiency leads to muscle membrane defects and apoptosis independent of dystrophin. Journal of Cell Biology $142,1279-1287$

63 Matsumura, K. et al. (2003) Disruption of dystroglycan axis by $\beta$-dystroglycan processing in cardiomyopathic hamster muscle. Neuromuscular Disorders 13, 796-803

64 Straub, V. et al. (1998) Molecular pathogenesis of muscle degeneration in the $\delta$-sarcoglycan-deficient hamster. American Journal of Pathology 153, 1623-1630

65 Hack, A.A. et al. (1999) Muscle degeneration without mechanical injury in sarcoglycan deficiency. Proceedings of the National Academy of Sciences of the United States of America 96, 10723-10728

66 Mizuno, Y. et al. (1995) Sarcoglycan complex is selectively lost in dystrophic hamster muscle. American Journal of Pathology 146, 530-536

67 Sasaoka, T. et al. (2003) Pathological analysis of muscle hypertrophy and degeneration in muscular dystrophy of $\gamma$-sarcoglycan deficient mice. Neuromuscular Disorders 13, 193-206

68 Greener, M.J. and Roberts, R.G. (2000) Conservation of components of the dystrophin complex in Drosophila. FEBS Letters 482, 13-18

69 Allikian, M.J. et al. (2007) Reduced life span with heart and muscle dysfunction in Drosophila sarcoglycan mutants. Human Molecular Genetics 16, 2933-2943

70 Chambers, S.P. et al. (2003) Sarcoglycans of the zebrafish: orthology and localization to the sarcolemma and myosepta of muscle. Biochemical and Biophysical Research Communications 303, 488-495

71 Cheng, L. et al. (2006) $\delta$-Sarcoglycan is necessary for early heart and muscle development in zebrafish. Biochemical and Biophysical Research Communications 344, 1290-1299

72 Ettinger, A.J., Feng, G. and Sanes, J.R. (1997) $\varepsilon$-Sarcoglycan, a broadly expressed homologue of the gene mutated in limb-girdle muscular dystrophy 2D. Journal of Biological Chemistry 272, 32534-32538

73 McNally, E.M., Ly, C.T. and Kunkel, J.M. (1998)

Human $\varepsilon$-sarcoglycan is highly related to $\alpha$-sarcoglycan (adhalin), the limb-girdle muscular dystrophy 2D gene. FEBS Letters 422, 27-32

74 Wheeler, M.T. et al. (2002) ל-sarcoglycan, a novel component of the sarcoglycan complex, is reduced in muscular dystrophy. Human Molecular Genetics 11, 2147-2154

75 Shiga, K. et al. (2006) $\zeta$-Sarcoglycan is a functional homologue of $\gamma$-sarcoglycan in the formation of the sarcoglycan complex. Experimental Cell Research 312, 2083-2092

76 Dickens, N.J., Beatons, S. and Ponting, C.P. (2004) Cadherin-like domains in $\alpha$-dystroglycan, $\alpha / \varepsilon$-sarcoglycan, yeast and bacterial proteins. Current Biology 12, 197-199

77 Zimprich, A. et al. (2001) Mutations in the gene encoding $\varepsilon$-sarcoglycan cause myoclonusdystonia syndrome. Nature Genetics 29, 66-69

78 Anastasi, G. et al. (2007) Sarcoglycan subcomplex in normal and pathological human muscle fibers. European Journal of Histochemistry 51 (Suppl. 1), 29-33

79 Ueda, H. et al. (2001) $\delta$ and $\gamma$-Sarcoglycan localization in the sarcoplasmic reticulum of skeletal muscle. Journal of Histochemistry and Cytochemistry 49, 529-537

80 Hegde, R.S. and Kang, S-W. (2008) The concept of translocational regulation. Journal of Cell Biology $182,225-232$

81 Caramelo, J.J. and Parodi, A.J. (2008) Getting in and out from calnexin/calreticulin cycles. Journal of Biological Chemistry 283, 10221-10225

82 Holt, K.H. and Campbell, K.P. (1998) Assembly of the sarcoglycan complex. Insights for muscular dystrophy. Journal of Biological Chemistry 273, 34667-34670

83 Noguchi, S. et al. (2000) Formation of sarcoglycan complex with differentiation in cultured myocytes. European Journal of Biochemistry 267, 640-648

84 Allikian, M.J. and McNally, E.M. (2007) Processing and assembly of the dystrophin glycoprotein complex. Traffic 8, 177-183

85 Shi, W. et al. (2004) Specific assembly pathway of sarcoglycans is dependent on $\beta$ - and $\delta$ sarcoglycan. Muscle and Nerve 29, 409-419

86 Draviam, R.A., Shand, S.H. and Watkins, S.C. (2006) The $\beta \delta$-core of sarcoglycan is essential for deposition at the plasma membrane. Muscle and Nerve 34, 691-701

Accession information: doi:10.1017/S1462399409001203; Vol. 11; e28; September 2009 (C) Cambridge University Press 2009. Re-use permitted under a Creative Commons Licence-by-nc-sa. 
87 Hack, A.A. et al. (2000) Differential requirement for individual sarcoglycans and dystrophin in the assembly and function of the dystrophinglycoprotein complex. Journal of Cell Science 113, 2535-2544

88 Draviam, R.A. et al. (2006) $\alpha$-Sarcoglycan is recycled from the plasma membrane in the absence of sarcoglycan complex assembly. Traffic 7, 1-18

89 Kobuke, K. et al. (2008) A common diseaseassociated missense mutation in $\alpha$-sarcoglycan fails to cause muscular dystrophy in mice. Human Molecular Genetics 17, 1201-1213

90 Loo, T.W. and Clarke, D.N. (2007) Chemical and pharmacological chaperones as new therapeutical agents. Expert Reviews in Molecular Medicine 9, 1-18

91 Fokkema, I.F., den Dunnen, J.T. and Taschner, P.E. (2005) LOVD: easy creation of a locus-specific sequence variation database using an "LSDB-in-abox" approach. Human Mutations 26, 63-68

92 Trabelsi, M. et al. (2008) Revised spectrum of mutations in sarcoglycanopathies. European Journal of Human Genetics 16, 793-803

93 Ferrer, I. and Olivè, M. (2008) Molecular pathology of myofibrillar myopathies. Expert Reviews in Molecular Medicine 10, e25

94 Olzmann, J.A., Li, L. and Chin, L.S. (2008) Aggresome formation and neurodegenerative diseases: therapeutic implications. Current Medicinal Chemistry 15, 47-60

95 Blom, N., Gammeltoft, S. and Brunak, S. (1999) Sequence- and structure-based prediction of eukaryotic protein phosphorylation sites. Journal of Molecular Biology 294, 1351-1362

96 Boito, C. et al. (2003) Novel sarcoglycan gene mutations in a large cohort of Italian patients. Journal of Medical Genetics 40, e67

97 Guglieri, M. et al. (2008) Clinical, molecular, and protein correlations in a large sample of genetically diagnosed Italian limb girdle muscular dystrophy patients. Human Mutation 29, 258-266

98 McNally, E.M. et al. (1996) Mutations that disrupt the carboxyl terminus of $\gamma$-sarcoglycan cause muscular dystrophy. Human Molecular Genetics 5, 1841-1847

99 Cossu, G. and Sampaolesi, M. (2007) New therapies for Duchenne muscular dystrophy: challenges, prospects and clinical trials. Trends in Molecular Medicine 13, 520-526

100 Danièle, N., Richard, I. and Bartoli, M. (2006) Ins and outs of therapy in limb girdle muscular dystrophies. International Journal of Biochemistry and Cell Biology 39, 1608-1624
101 Lim, L.E. and Rando, T.A. (2008) Technology insight: therapy for Duchenne muscular dystrophy-an opportunity for personalized medicine? Nature Clinical Practice Neurology 4, 149-158

102 Allamand, V. et al. (2000) Early adenovirusmediated gene transfer effectively prevents muscular dystrophy in $\alpha$-sarcoglycan-deficient mice. Gene Therapy 7, 1385-1391

103 Fougerousse, F. et al. (2007) Phenotypic correction of $\alpha$-sarcoglycan deficiency by intra-arterial injection of a muscle-specific serotype $1 \mathrm{rAAV}$ vector. Molecular Therapy 15, 53-61

104 Xiao, X. et al. (2000) Full functional rescue of a complete muscle (TA) in dystrophic hamsters by adeno-associated virus vector-directed gene therapy. Journal of Virology 74, 1436-1442

105 Price, F.D. et al. (2007) Stem cell based therapies to treat muscular dystrophy. Biochimica et Biophysica Acta 1772, 272-283

106 Lapidos, K.A. et al. (2004) Transplanted hematopoietic stem cells demonstrate impaired sarcoglycan expression after engraftment into cardiac and skeletal muscle. Journal of Clinical Investigation 114, 1577-1585

107 Wallace, G.Q. et al. (2008) Long-term survival of transplanted stem cells in immunocompetent mice with muscular dystrophy. American Journal of Pathology 173, 792-802

108 Sampaolesi, M. et al. (2003) Cell therapy of $\alpha$-sarcoglycan null dystrophic mice through intra-arterial delivery of mesoangioblasts. Science 301, 487-492

109 Sampaolesi, M. et al. (2006) Mesoangioblast stem cells ameliorate muscle function in dystrophic dogs. Nature 444, 574-579

110 Hermann, T. (2007) Aminoglycoside antibiotics: old drugs and new therapeutic approaches.

Cellular and Molecular Life Sciences 64, 1841-1852

111 Barton-Davis, E.R. et al. (1999) Aminoglycoside antibiotics restore dystrophin function to skeletal muscles of mdx mice. Journal of Clinical Investigation 104, 375-381

112 Dunant, P. et al. (2003) Gentamicin fails to increase dystrophin expression in dystrophin-deficient muscle. Muscle and Nerve 27, 624-627

113 Du, M. et al. (2008) PTC124 is an orally bioavailable compound that promotes suppression of the human CFTR-G542X nonsense allele in a CF mouse model. Proceedings of the National Academy of Sciences of the United States of America 105, 2064-2069

114 Welch, E.M. et al. (2007) PTC124 targets genetic disorders caused by nonsense mutations. Nature $447,87-91$ 
115 van Ommen, G.J., van Deutekom, J. and AartsmaRus, A. (2008) The therapeutic potential of antisense-mediated exon skipping. Current Opinion in Molecular Therapeutics 10, 140-149

116 Serose, A. et al. (2006) Short-term treatment using insulin-like growth factor-1 (IGF-1) improves life expectancy of the $\delta$-sarcoglycan deficient hamster. Journal of Gene Medicine 8, 1048-1055

117 Minetti, G.C. et al. (2006) Functional and morphological recovery of dystrophic muscles in mice treated with deacetylase inhibitors. Nature Medicine 12, 1147-1150

118 Bartoli, M. et al. (2007) AAV-mediated delivery of a mutated myostatin propeptide ameliorates calpain 3 but not $\alpha$-sarcoglycan deficiency. Gene Therapy 14, 733-740

119 Bogdanovich, S., McNally, E.M. and Khurama, T.S. (2008) Myostatin blockade improves function but not histopathology in a murine model of limbgirdle muscular dystrophy 2C. Muscle and Nerve 37, 308-316

120 Vembar, S.S. and Brodsky, J.L. (2008) One step at a time: endoplasmic reticulum-associated degradation. Nature Reviews. Molecular Cell Biology 9, 944-957

121 Vashist, S. and Ng, D.T.W. (2004) Misfolded proteins are sorted by a sequential checkpoint mechanism of ER quality control. Journal of Cell Biology 165, 41-52

122 Christianson, J.C. et al. (2008) OS-9 and GRP94 deliver mutant a1-antitrypsin to the Hrd1-SEL1L ubiquitin ligase complex for ERAD. Nature Cell Biology 10, 272-282

123 Nakatsukasa, K. and Brodsky, J.L. (2008) The recognition and retrotranslocation of misfolded proteins from the endoplasmic reticulum. Traffic 9, 861-870

124 Romish, K. (2006) Cdc48p is UBX-linked to ER ubiquitin ligases. Trends in Biochemical Sciences 31, 24-25

125 Suzuki, T. (2007) Cytoplasmic peptide:N-glycanase and catabolic pathway for free N-glycans in the cytosol. Seminars in Cell and Developmental Biology 18, 762-769

126 Ron, D. and Walter, P. (2007) Signal integration in the endoplasmic reticulum unfolded protein response. Nature Reviews. Molecular Cell Biology $8,519-529$

127 Jessop, C.E. et al. (2007) ERp57 is essential for efficient folding of glycoproteins sharing common structural domains. EMBO Journal 26, 28-40

128 Gaucci, E. et al. (2008) The binding of antibiotics to ERp57/GRP58. Journal of Antibiotics 61, 400-402
129 Gelman, M.S. and Kopito, R.R. (2003) Cystic fibrosis: premature degradation of mutated proteins as a molecular disease mechanism. Methods in Molecular Biology 232, 27-37

130 Turnbull, E.L. (2007) The role of UPS in cystic fibrosis. BMC Biochemistry 8 (Suppl. I), S11

131 Norez, C. et al. (2008) Proteasome-dependent pharmacological rescue of cystic fibrosis transmembrane conductance regulator revealed by mutation of glycine 622. Journal of Pharmacology and Experimental Therapeutics 325, 89-99

132 Vij, N., Fang, S. and Zeritlin, P.L. (2006) Selective inhibition of endoplasmic reticulum-associated degradation rescues $\Delta$ F508-cystic fibrosis transmembrane regulator and suppresses interleukin-8 levels. Journal of Biological Chemistry 281, 17369-17378

133 Fuller, W. and Cuthbert, A.W. (2000) Posttranslational disruption of the $\Delta \mathrm{F} 508$ cystic fibrosis transmembrane conductance regulator (CFTR)molecular chaperone complex with geldanamycin stabilizes $\Delta$ F508 CFTR in the rabbit reticulocyte lysate. Journal of Biological Chemistry 275, 37462-37468

134 Sun, F. et al. (2006) Derlin-1 promotes the efficient degradation of the cystic fibrosis transmembrane conductance regulator (CFTR) and CFTR folding mutants. Journal of Biological Chemistry 281, 36856-36863

135 Weihl, C.C. et al. (2007) Transgenic expression of inclusion body myopathy associated mutant p97/ VCP causes weakness and ubiquitinated protein inclusions in mice. Human Molecular Genetics 16, 919-928

136 Wang, Q., Li, L. and Ye, Y. (2008) Inhibition of p97-dependent protein degradation by Eeyarestatin I. Journal of Biological Chemistry 283, 7445-7454

137 Hagihara, S. et al. (2007) Fluorescently labeled inhibitor for profiling cytoplasmic peptide-Nglycanase. Glycobiology 17, 1070-1076

138 Misaghi, S. et al. (2004) Using a small molecule inhibitor of peptide:N-glycanase to probe its role in glycoprotein turnover. Chemistry and Biology 11, 1677-1687

139 Horibe, T. et al. (2001) Ribostamycin inhibits the chaperone activity of protein disulfide isomerase. Biochemical and Biophysical Research Communications 289, 967-972

140 Bonuccelli, G. et al. (2003) Proteasome inhibitor (MG-132) treatment of mdx mice rescues the expression and membrane localization of dystrophin and dystrophin-associated 
proteins. American Journal of Pathology 163, 1663-1675

141 Bonucelli, G. et al. (2007) Localized treatment with a novel FDA-approved proteasome inhibitor blocks the degradation of dystrophin and dystrophin-associated proteins in mdx mice. Cell Cycle 6, 1242-1248

142 Assereto, S. et al. (2006) Pharmaceutical rescue of the dystrophin complex in Duchenne and Becker skeletal muscle explants by proteasomal inhibitor treatment. American Journal of Physiology 290, C577-582

143 Esapa, C.T. et al. (2007) SGCE missense mutations that causes myoclonus-dystonia syndrome impair e-sarcoglycan trafficking to the plasma membrane: modulation by polyubiquitination and torsinA. Human Molecular Genetics 16, 327-342

\section{Further reading, resources and contacts}

\section{Publications}

Straub, V. and Bushby, K. (2008) Therapeutic possibilities in the autosomal recessive limb-girdle muscular dystrophies. Neurotherapeutics 5, 619-626

This review describes an overview of potential novel therapeutical interventions in autosomal recessive limb-girdle muscular dystrophies.

Muir, L.A. and Chamberlain, J.S. (2009) Emerging strategies for cell and gene therapy of the muscular dystrophies. Expert Reviews in Molecular Medicine 11, e8

This review describes novel therapeutical interventions developed to cure Duchenne muscular dystrophy and that can be extended to other dystrophies.

Linde, L. and Kerem, B. (2008) Introducing sense into nonsense in treatments of human genetic diseases. Trends in Genetics 24, 552-563

This review deals with therapeutical approaches aimed at curing nonsense mutations creating a premature stop codon.

Braun, S. (2008) Muscular gene transfer using nonviral vectors. Current Gene Therapy 8, 391-405

This review reports on recent progresses of plasmid delivery of genes to muscles by using non-viral vectors.

\section{Websites}

An up-to-date list of allelic variants of sarcoglycan genes is available at the Leiden Open Variation Database (LOVD) gene homepage, created by Johan den Dunnen and colleagues at Leiden University Medical Center: http://www.dmd.nl/sgca_seqvar.html

The Muscular Dystrophy Association, MDA, together with numerous international associates, provides information on muscular dystrophies and helpful resources for patients: http://www.mda.org/

Treat Neuromuscular Disorders (TREAT-NMD) provide ample information about muscular dystrophies and updates on new therapeutic developments:

http://www.treat-nmd.eu/home.php

The Center for Neuromuscular Diseases, CNMD, is dedicated to clinical trials and therapy: http://www.cnmd.ac.uk/index.html

ClinicalTrialsGov offers a complete list of worldwide clinical trials on muscular dystrophies, and other medical conditions:

http://clinicaltrials.gov/ct2/home 


\section{Figures}

Figure 1. The dystrophin-glycoprotein complex.

Figure 2. Trafficking of the sarcoglycan complex.

Figure 3. Putative ER processing of wild-type and mutant sarcoglycans.

Figure 4. Putative progression of sarcoglycan mutants through the ER-associated degradation pathway.

Figure 5. Sarcoglycan missense mutations.

\section{Tables}

Table 1. Human sarcoglycan protein data.

Table 2. ER quality control and ERAD targets for possible therapy.

\section{Citation details for this article}

Dorianna Sandonà and Romeo Betto (2009) Sarcoglycanopathies: molecular pathogenesis and therapeutic prospects. Expert Rev. Mol. Med. Vol. 11, e28, September 2009, doi:10.1017/S1462399409001203 